H. Fujimoto

Nagoya Math. J.

Vol. 64 (1976), 117-147

\title{
A UNIQUENESS THEOREM OF ALGEBRAICALLY NON-DEGENERATE MEROMORPHIC MAPS INTO $P^{N}(C)$
}

\author{
HIROTAKA FUJIMOTO
}

\section{§1. Introduction.}

In the previous paper [3], the author generalized the uniqueness theorems of meromorphic functions given by G. Pólya in [5] and $R$. Nevanlinna in [4] to the case of meromorphic maps of $C^{n}$ into the $N$ dimensional complex projective space $P^{N}(C)$. He studied two meromorphic maps $f$ and $g$ of $C^{n}$ into $P^{N}(C)$ such that, for $q$ hyperplanes $H_{i}$ in $P^{N}(C)$ with $f\left(C^{n}\right) \varnothing H_{i}, g\left(C^{n}\right) \varangle H_{i}$ located in general position, the pull-backs $\nu\left(f, H_{i}\right)$ and $\nu\left(g, H_{i}\right)$ of divisors $\left(H_{i}\right)$ on $P^{N}(C)$ by $f$ and $g$ are equal to each other. Under some additional assumptions, he revealed the existence of some special types of relations between $f$ and $g$. For example, he showed that, if $f$ or $g$ is non-degenerate, namely, the image is not included in any hyperplane in $P^{N}(C)$ and $q=3 N+2$, then $f \equiv g$.

We consider in this paper meromorphic maps into $P^{N}(C)$ which are algebraically non-degenerate, namely, whose images are not included in any proper subvariety of $P^{N}(C)$. We give the following theorem.

THEOREM. Let $f, g$ be meromorphic maps of $C^{n}$ into $P^{N}(C)$ such that $\nu\left(f, H_{i}\right)=\nu\left(g, H_{i}\right)$ for $2 N+3$ hyperplanes $H_{i}$ located in general position. If $f$ or $g$ is algebraically non-degenerate, then $f \equiv g$.

To show this, after giving some preliminaries ( $\$ 2)$, we provide in $\S 3$ some combinatorial lemmas which act essential roles in this paper. A main one of them is proved in $\S 4$. And, in $\S 5$, the smallest algebraic set $V_{f, g}$ in $P^{N}(C)$ which includes the set $(f \times g)\left(C^{n}\right)$ is studied in the case that $2 N+2$ hyperplanes $H_{i}$ with $\nu\left(f, H_{i}\right)=\nu\left(g, H_{i}\right)$ are given. It is shown that $V_{f, g}$ is an at most $N$-dimensional irreducible algebraic set.

Received March 12, 1976. 
After these preparations, we prove the above theorem in $\S 6$. We show also the existence of some special types of relations between algebraically non-degenerate meromorphic maps $f$ and $g$ such that $\nu\left(f, H_{i}\right)=\nu\left(g, H_{i}\right)$ for $2 N+2$ hyperplanes $H_{i}$ in general position. In the last section, we study meromorphic maps into $P^{2}(C)$ or $P^{3}(C)$ more precisely. For the above meromorphic maps $f$ and $g$, it is shown that they are related as $L \cdot g=f$ with a special type of projective linear transformation $L$ of $P^{N}(C)$ in the case $N=2$ and the algebraic set $V_{f, g}$ is included in an algebraic set defined by some special types of equations of degree at most two in the case $N=3$.

\section{§2. Preliminaries.}

2.1. We shall recall some notations and results in the previous paper [3].

Let $f$ be a meromorphic map of $C^{n}$ into $P^{N}(C)$. For arbitrarily fixed homogeneous coordinates $w_{1}: w_{2}: \cdots: w_{N+1}$ on $P^{N}(C)$, we can find holomorphic functions $f_{1}(z), \ldots, f_{N+1}(z)$ on $C^{n}$ such that the analytic set

$$
I(f):=\left\{z \in C ; f_{1}(z)=\cdots=f_{N+1}(z)=0\right\}
$$

is of codimension at least two and $f$ is represented as

$$
f(z)=f_{1}(z): f_{2}(z): \cdots: f_{N+1}(z) \quad\left(z \in C^{n}-I(f)\right) .
$$

In the following, we shall call such a representation an admissible representation of $f$ on $C^{n}$. As is easily seen, for two admissible representations

$$
f=f_{1}: f_{2}: \cdots: f_{N+1}=\tilde{f}_{1}: \tilde{f}_{2}: \cdots: \tilde{f}_{N+1}
$$

of $f, \tilde{f}_{1} / f_{1}\left(=\tilde{f}_{i} / f_{i}(2 \leqq i \leqq N+1)\right)$ is a nowhere zero holomorphic function on $C^{n}$. For a given hyperplane

$$
H: a^{1} w_{1}+a^{2} w_{2}+\cdots+a^{N+1} w_{N+1}=0
$$

in $P^{N}(C)$ with $f\left(C^{n}\right) \measuredangle H$, we define a holomorphic function

$$
F_{f}^{H}:=a^{1} f_{1}+\cdots+a^{N+1} f_{N+1}
$$

with an admissible representation $f=f_{1}: f_{2}: \cdots: f_{N+1}$ on $C^{n}$ and denote by $\nu(f, H)(a)$ the zero multiplicity of $F_{f}^{H}$ at a point $a \in C^{n}$, which is uniquely determined independently of any choices of homogeneous coordinates and admissible representations. 
Now, let us consider two non-constant meromorphic maps $f$ and $g$ of $C^{n}$ into $P^{N}(C)$ and $q(\geqq 2 N+2)$ hyperplanes

$$
H_{i}: a_{i}^{1} w_{1}+a_{i}^{2} w_{2}+\cdots+a_{i}^{N+1} w_{N+1}=0 \quad(1 \leqq i \leqq q)
$$

in $P^{N}(C)$ located in general position. We shall study these maps under the assumption that $f\left(C^{n}\right) \varnothing H_{i}, g\left(C^{n}\right) \varnothing H_{i}$ and $\nu\left(f, H_{i}\right)=\nu\left(g, H_{i}\right)$ for any $i$. We define functions

$$
h_{i}:=F_{f}^{H_{i}} / F_{g}^{H_{i}}
$$

with holomorphic functions $F_{f}^{H_{i}}$ and $F_{g}^{H_{i}}$ defined as (2.2) for arbitrarily fixed admissible representations of $f$ and $g$. By the assumption, each $h_{i}$ is a nowhere zero holomorphic function on $C^{n}$ and the ratios $h_{i} / h_{j}$ are uniquely determined independently of any choices of homogeneous coordinates, representations (2.3) of $H_{i}$ and admissible representations of $f$ and $g$.

For the case $q=2 N+2$, by eliminating $f_{1}, \cdots, f_{N+1}, g_{1}, \cdots, g_{N+1}$ from the identities

$$
a_{i}^{1} f_{1}+\cdots+a_{i}^{N+1} f_{N+1}=h_{i}\left(a_{i}^{1} g_{1}+\cdots+a_{i}^{N+1} g_{N+1}\right),
$$

we obtain a relation

$$
\operatorname{det}\left(a_{i}^{1}, \cdots, a_{i}^{N+1}, h_{i} a_{i}^{1}, \cdots, h_{i} a_{i}^{N+1} ; 1 \leqq i \leqq 2 N+2\right)=0 .
$$

Then, by the Laplace' expansion formula, we can show easily

(2.6) Among holomorphic functions $h_{i}$ satisfying the relation (2.5) there is a relation of the type

$$
\sum_{1 \leq i_{1}<\cdots<i_{N+1} \leqq 2 N+2} A_{i_{1} \cdots i_{N+1}} h_{i_{1}} h_{i_{2}} \cdots h_{i_{N+1}}=0,
$$

where $A_{i_{1} \ldots i_{N+1}}$ are non-zero constants (cf., [3], Proposition 3.5).

2.2. Let $H^{*}$ be the multiplicative group of all nowhere zero holomorphic functions on $C^{n}$. We may regard the set $C^{*}=C-\{0\}$ as a subgroup of $\boldsymbol{H}^{*}$. Then, the factor group $G:=\boldsymbol{H}^{*} / \boldsymbol{C}^{*}$ is a torsionfree abelian group. We denote by $[h]$ the class in $G$ containing an element $h$ in $\boldsymbol{H}^{*}$. For two elements $h, h^{*} \in \boldsymbol{H}^{*}$, by the notation $h \sim h^{*}$ we mean $[h]=\left[h^{*}\right]$ in $G$.

As an easy consequence of the classical theorem of E. Borel, we know the following fact ([1], [2] and [3], Remark to Corollary 4.2). 
(2.7) Let $h_{1}, \cdots, h_{p} \in \boldsymbol{H}^{*}$ satisfy the relation

$$
a^{1} h_{1}+a^{2} h_{2}+\cdots+a^{p} h_{p}=0
$$

for some $a^{i} \in C^{*}$. Then, for any $h_{i}$, there exists some $h_{j}(i \neq j)$ such that $h_{i} \sim h_{j}$.

By (2.6) and (2.7), we can conclude

(2.8) Let $\alpha_{1}, \alpha_{2}, \cdots, \alpha_{2 N+2}$ be elements in $\boldsymbol{H}^{*} / C^{*}$. Assume that (2.5) holds for suitable $h_{i} \in H^{*}$ with $\alpha_{i}=\left[h_{i}\right]$ and $a(2 N+2) \times(N+1)$ matrix $A=\left(a_{i}^{j}\right)$ whose minors of degree $N+1$ do not vanish. Then, for any $i_{1}, \cdots, i_{N+1}\left(1 \leqq i_{1}<\cdots<i_{N+1} \leqq 2 N+2\right)$, there exist some $j_{1}, \cdots, j_{N+1}$ with $1 \leqq j_{1}<\ldots<j_{N+1} \leqq 2 N+2$ and $\left\{i_{1}, \cdots, i_{N+1}\right\} \neq$ $\left\{j_{1}, \cdots, j_{N+1}\right\}$ such that

$$
\alpha_{i_{1}} \alpha_{i_{2}} \cdots \alpha_{i_{N+1}}=\alpha_{j_{1}} \alpha_{j_{2}} \cdots \alpha_{j_{N+1}}
$$

And, we have also

(2.9) Let $h_{1}, h_{2}, \cdots, h_{t}$ be elements in $\boldsymbol{H}^{*}$ such that $h_{1}^{\ell_{1}} h_{2}^{\ell_{2}} \cdots h_{t}^{\ell_{t}} \notin \boldsymbol{C}^{*}$ for any integers $\left(\ell_{1}, \cdots, \ell_{t}\right)(\neq(0, \cdots, 0))$. Then, for any not identically zero polynomial $P\left(X_{1}, \cdots, X_{t}\right), P\left(h_{1}, \cdots, h_{t}\right)$ does not vanish identically.

For the proof, see Proposition 4.5 in [3].

\section{§3. Combinatorial lemmas.}

3.1. Let $G$ be a torsionfree abelian group. Take a $q$-tuple $A=\left(\alpha_{1}\right.$, $\left.\cdots, \alpha_{q}\right)$ of elements $\alpha_{i}$ in $G$. We denote by $\left\{\left\{\alpha_{1}, \cdots, \alpha_{q}\right\}\right\}$, or simply $\tilde{A}$, the subgroup of $G$ generated by $\alpha_{1}, \cdots, \alpha_{q}$ and $t(A)$ the rank of $\tilde{A}$, where $t(A)=0$ means $\alpha_{1}=\cdots=\alpha_{q}=1$ (=the unit elements of $G$ ). It has a basis $\beta_{1}, \cdots, \beta_{t}(t=t(A))$ and each $\alpha_{i}$ is uniquely represented as

$$
\alpha_{i}=\beta_{1}^{\ell_{i 1}} \beta_{2}^{\ell_{22}} \cdots \beta_{t}^{\ell_{i t}}
$$

with suitable integers $\ell_{i_{\tau}}$. We may regard $G$ as a subgroup of $G \otimes_{Z} \boldsymbol{Q}$, where $\boldsymbol{Z}$ and $\boldsymbol{Q}$ denote the additive groups of all integers and of all rational numbers respectively. Then, we can choose some $\alpha_{i_{1}}, \cdots, \alpha_{i_{t}}$ among $\alpha_{1}, \cdots, \alpha_{q}$ as a basis of the subgroup of $G \otimes_{Z} \boldsymbol{Q}$ generated by $\alpha_{1}, \cdots, \alpha_{q}$ as a $\boldsymbol{Q}$-module.

(3.2) There exists a basis $\left\{\beta_{1}, \cdots, \beta_{t}\right\}$ of $\left\{\left\{\alpha_{1}, \cdots, \alpha_{q}\right\}\right\}$ in $G$ such that, for 
suitable $i_{1}, \cdots, i_{t}$ and non-zero integers $\ell_{\tau}, \beta_{\tau}^{\ell_{\tau}}=\alpha_{i_{\tau}}$, namely, $\ell_{i_{\tau^{\sigma}}}=0$ $(\sigma \neq \tau)$ in the representation (3.1).

In the followings, we shall call a basis of $\tilde{A}$ with the property as in (3.2) to be an adequate basis for $\tilde{A}$.

For convenience' sake, we introduce some notations. For the set $I_{r}:=\{1,2, \cdots, r\}$, we mean by a combination $\left(\left(i_{1}, \cdots, i_{s}\right)\right)$ in $I_{r}$ the set of integers $i_{1}, \cdots, i_{s}$ with $1 \leqq i_{1}<\ldots<i_{s} \leqq r$. And, we indicate by $\widetilde{\Im}_{r, s}$ the set of all combinations of $s$ elements in $I_{r}$. For an arbitrarily fixed $r$-tuple $A=\left(\alpha_{1}, \cdots, \alpha_{r}\right)$ of elements in $G$, we use an abbreviated notation

$$
A_{I}=\alpha_{i_{1}} \alpha_{i_{2}} \cdots \alpha_{i_{s}}
$$

when $I=\left(\left(i_{1}, i_{2}, \cdots, i_{s}\right)\right) \in \widetilde{S}_{r, s}$.

Definition 3.3. Let $q \geqq r>s \geqq 1$. A $q$-tuple $A=\left(\alpha_{1}, \alpha_{2}, \cdots, \alpha_{q}\right)$ of elements in $G$ is called to have the property $\left(P_{r, s}\right)$ if any chosen $r$-tuple $A^{\prime}=\left(\alpha_{k_{1}}, \cdots, \alpha_{k_{r}}\right) \quad\left(1 \leqq k_{1}<\cdots<k_{r} \leqq q\right)$, put $A^{\prime}:=\left(\alpha_{1}^{\prime}, \cdots, \alpha_{r}^{\prime}\right)=\left(\alpha_{k_{1}}\right.$, $\left.\cdots, \alpha_{k_{r}}\right)$, satisfies the condition that for any $I$ in $\mathfrak{\Im}_{r, s}$ there exists some $J$ in $\widetilde{S}_{r, s}$ with $I \neq J$ such that

$$
A_{I}^{\prime}=A_{J}^{\prime}
$$

Let $A=\left(\alpha_{1}, \cdots, \alpha_{q}\right)$ be a $q$-tuple of elements in $G$ with the property $\left(P_{r, s}\right)$. To study relations among $\alpha_{i}$, we choose a basis $\beta_{1}, \cdots, \beta_{t}$ for which each $\alpha_{i}$ is represented as (3.1). Then, we can find integers $p_{1}$, $\cdots, p_{t}$ such that, when we put

$$
\ell_{i}:=\ell_{i 1} p_{1}+\ell_{i 2} p_{2}+\cdots+\ell_{i t} p_{t} \quad(1 \leqq i \leqq q),
$$

$\ell_{i}=\ell_{j}$ holds only if

$$
\left(\ell_{i 1}, \ell_{i 2}, \cdots, \ell_{i t}\right)=\left(\ell_{j 1}, \ell_{j 2}, \cdots, \ell_{j t}\right),
$$

(cf., [3], (2.2)).

LEMMA 3.4. In the above situation, if the indices $i$ of $\alpha_{i}$ are chosen so that

$$
\ell_{1} \leqq \ell_{2} \leqq \cdots \leqq \ell_{q}
$$

then

$$
\ell_{s}=\ell_{s+1}=\cdots=\ell_{q+s-r+1}
$$


and so

$$
\alpha_{s}=\alpha_{s+1}=\cdots=\alpha_{q+s-r+1} .
$$

For the proof, see Lemma 2.6 in [3].

Since $q+s-r+1 \geqq s+1 \geqq 2$ in any case, we have

LEMMA 3.5. For any $q$-tuple $A=\left(\alpha_{1}, \cdots, \alpha_{q}\right)$, if $A$ has the property $\left(P_{r, s}\right)(1 \leqq s<r \leqq q)$, there exist two distinct indices $i, j$ such that $\alpha_{i}=\alpha_{j}$.

3.2. Let us introduce another notation. For elements $\alpha_{1}, \alpha_{2}, \cdots, \alpha_{q}$, $\alpha_{1}^{*}, \alpha_{2}^{*}, \cdots, \alpha_{q}^{*}$ in $G$, by the notation

$$
\alpha_{1}: \alpha_{2}: \cdots: \alpha_{q}=\alpha_{1}^{*}: \alpha_{2}^{*}: \cdots: \alpha_{q}^{*}
$$

we mean that $\alpha_{i}=\beta \alpha_{i}^{*}(1 \leqq i \leqq q)$ for some $\beta \in G$.

Now, we give the following main lemma.

LEMMA 3.6. Let $1 \leqq s<q \leqq 2 s$ and $A=\left(\alpha_{1}, \cdots, \alpha_{q}\right)$ be a $q$-tuple elements in $G$ with the property $\left(P_{q, s}\right)$ and assume $\alpha_{i}=1$ for some $i$. Then,

(i) the rank $t(A)$ of $\left\{\left\{\alpha_{1}, \cdots, \alpha_{q}\right\}\right\}$ is not larger than $s-1$,

(ii) if $t(A)=s-1, q=2 s$ and $a$ basis $\beta_{1}, \cdots, \beta_{s-1}$ of $\left\{\left\{\alpha_{1}, \cdots, \alpha_{q}\right\}\right\}$ can be chosen so that, after suitable changes of indices, $\alpha_{1}, \cdots, \alpha_{q}$ are represented as one of the following two types;

(A) $s$ is odd and

$$
\alpha_{1}: \alpha_{2}: \cdots: \alpha_{2 s}=1: 1: \beta_{1}: \beta_{1}: \beta_{2}: \beta_{2}: \cdots: \beta_{s-1}: \beta_{s-1}
$$

(B) $\alpha_{1}: \alpha_{2}: \cdots: \alpha_{2 s}$

$$
\begin{aligned}
=1: & \cdots: 1: \beta_{1}: \cdots: \beta_{s-1}:\left(\beta_{1} \cdots \beta_{a_{1}}\right)^{-1}:\left(\beta_{a_{1}+1} \cdots \beta_{a_{2}}\right)^{-1}: \cdots \\
& \cdots:\left(\beta_{a_{k-1}+1} \cdots \beta_{a_{k}}\right)^{-1},
\end{aligned}
$$

where $0 \leqq k \leqq s-1, a_{k}-a_{k-1} \leqq s-k$ (put $a_{0}=0$ ) for any $\kappa$ and the unit element 1 appears $s-k+1$ times in the right hand side.

The proof of Lemma 3.6 will be given in the next section.

3.3. We shall show here that $A=\left(\alpha_{1}, \cdots, \alpha_{2 s}\right)$ of the type (A) or (B) of Lemma 3.6 satisfies actually the condition $\left(P_{2 s, s}\right)$.

Let us consider first $A=\left(\alpha_{1}, \cdots, \alpha_{2 s}\right)$ of the type (A). Since $s$ is odd, for any given combination $I=\left(\left(i_{1}, \cdots, i_{s}\right)\right) \in \mathfrak{J}_{2 s, s}$ we can find some $\alpha_{\tau_{0}}$ with $1 \leqq \tau_{0} \leqq s$ such that one of $\alpha_{2 \tau_{0}}$ and $\alpha_{2 \tau_{0}+1}$ equals some $a_{i_{\tau}}$ and 
the other does not equal any $\alpha_{i_{1}}, \alpha_{i_{2}}, \cdots, \alpha_{i_{s}}$. Exchanging indices if necessary, we may assume $2 \tau_{0}=i_{\tau}$ and $2 \tau_{0}+1 \neq i_{1}, \cdots, i_{s}$. Then, if we put $J:=\left(\left(i_{1}, \cdots, i_{\tau-1}, 2 \tau_{0}+1, i_{\tau+1}, \cdots, i_{s}\right)\right)\left(\in \widetilde{S}_{2 s, s}\right)$, it satisfies the conditions $I \neq J$ and $A_{I}=A_{J}$. This shows that $A$ has the property $\left(P_{2 s, s}\right)$.

We study next $A=\left(\alpha_{1}, \cdots, \alpha_{2 s}\right)$ of the type (B). Take an arbitrary combinations $I=\left(\left(i_{1}, \cdots, i_{s}\right)\right) \in \widetilde{\Im}_{2 s, s}$. Firstly, we consider the case $\left\{i_{1}\right.$, $\left.\cdots, i_{s}\right\} \cap\{1,2, \cdots, s-k+1\} \neq \phi$. If $\quad\{1,2, \cdots, s-k+1\} \varnothing\left\{i_{1}, \cdots, i_{s}\right\}$, for example, $i_{1}=1, i_{2} \neq 2$, then a combination $J=\left(\left(2, i_{2}, \cdots, i_{s}\right)\right)$ satisfies the conditions $I \neq J$ and $A_{I}=A_{J}$. We assume now $\{1,2, \cdots, s-k+1\}$ $\subseteq\left\{i_{1}, \cdots, i_{s}\right\}$. Let

$$
\begin{aligned}
& i_{1}=1, \cdots, i_{s-k+1}=s-k+1<i_{s-k+2}<\cdots \\
& \ldots<i_{\ell} \leqq 2 s-k<i_{\ell+1}<\ldots<i_{s} \leqq 2 s
\end{aligned} .
$$

Then, there exists some $\alpha_{i_{0}}\left(i_{0} \geqq 2 s-k+1\right)$ with the expression

$$
\alpha_{i_{0}}=\left(\beta_{a_{x+1}} \beta_{a_{k}+2} \cdots \beta_{a_{k+1}}\right)^{-1}
$$

for some $k(0 \leqq k \leqq k-1)$ such that $\alpha_{i_{0}} \neq \alpha_{i_{\ell+1}}, \cdots, \alpha_{i_{s}}$ and $\beta_{\sigma} \neq \alpha_{i_{s-k+2}}$, $\cdots, \alpha_{i_{\ell}}$ for any $\sigma\left(a_{k}+1 \leqq \sigma \leqq a_{x+1}\right)$. In fact, if not, at least one $\beta_{z}$ among $\alpha_{i_{s-k+2}}, \cdots, \alpha_{i_{\ell}}$ is used to express each $\alpha_{i}(i \geqq 2 s-k+1)$ with $\alpha_{i} \neq \alpha_{i_{\ell+1}}$, $\cdots, \alpha_{i_{s}}$ as (3.1) and so at least $k-(s-\ell)$ elements in $\left\{\alpha_{i_{s-k+2}}, \cdots, \alpha_{i_{\ell}}\right\}$ are necessary. But, the number of elements $\alpha_{i_{s-k+2}}, \cdots, \alpha_{i_{\ell}}$ is only $k-$ $s+\ell-1$. Therefore, we can choose a suitable $\alpha_{i_{0}}$ satisfying the desired condition. Then, since $a_{x+1}-a_{k} \leqq s-k$,

$$
\begin{aligned}
\alpha_{i_{1}} \alpha_{i_{2}} \cdots \alpha_{i_{s}} & =\alpha_{1} \alpha_{2} \cdots \alpha_{s-k+1} \alpha_{i_{s-k+2}} \cdots \alpha_{i_{s}} \\
& =\alpha_{1} \cdots \alpha_{s-k-a_{k+1}+a_{k}} \alpha_{i_{0}} \beta_{a_{k+1}} \cdots \beta_{a_{k+1} \alpha_{i_{s-k+2}} \cdots \alpha_{i_{s}} .}
\end{aligned}
$$

If we define a combination $J=\left(\left(j_{1}, \cdots, j_{s}\right)\right) \in \widetilde{S}_{2 s, s}$ so that

$$
\left\{\alpha_{1}, \cdots, \alpha_{s-k-a_{k+1}+a_{k}}, \alpha_{i_{0}}, \beta_{a_{k}+1}, \cdots, \beta_{a_{k+1}}, \alpha_{i_{s-k+2}}, \cdots, \alpha_{i_{s}}\right\}=\left\{\alpha_{j_{1}}, \alpha_{j_{2}}, \cdots \alpha_{j_{s}}\right\},
$$

it satisfies the conditions $I \neq J$ and $A_{I}=A_{J}$.

It remains to examine the case $\{1,2, \cdots, s-k+1\} \cap\left\{i_{1}, \cdots, i_{s}\right\}=\phi$.

Let us assume

$$
s-k+1<i_{1}<\ldots<i_{\ell} \leqq 2 s-k<i_{\ell+1}<\ldots<i_{s} \leqq 2 s .
$$

Then, there exists some $\alpha_{i_{\tau_{0}}}\left(\ell+1 \leqq \tau_{0} \leqq s\right)$ such that

$$
\alpha_{i_{\tau_{0}}}=\left(\beta_{a_{\kappa^{\prime}+1}} \cdots \beta_{a_{\kappa^{\prime}+1}}\right)^{-1}
$$


for a suitable $\kappa^{\prime}\left(0 \leqq \kappa^{\prime} \leqq k-1\right)$ and each $\beta_{\sigma}\left(a_{\varepsilon^{\prime}}+1 \leqq \sigma \leqq a_{k^{\prime}+1}\right)$ coincides with $\alpha_{i_{\tau}}(1 \leqq \tau \leqq \ell)$. In fact, if not, for each $\alpha_{i}$ of $\alpha_{i_{\ell+1}}, \cdots, \alpha_{i_{s}}$ some $\beta_{\sigma}$ with $\beta_{\sigma} \notin\left\{\alpha_{i_{1}}, \cdots, \alpha_{i_{\ell}}\right\}$ appears in the expression of $\alpha_{i}$ as (3.1). But, there are only $s-\ell-1 \beta_{\sigma}$ with $\beta_{\sigma} \neq \alpha_{i_{1}}, \cdots, \alpha_{i_{\ell}}$. So, a suitable $\alpha_{i_{\tau_{0}}}$ has the desired property. Then, if we define a combination $J=\left(\left(j_{1}^{\prime}\right.\right.$, $\left.\left.\cdots, j_{s}^{\prime}\right)\right) \in \widetilde{\mathfrak{N}}_{2 s, s}$ so that

$$
\left\{\alpha_{1}, \cdots, \alpha_{a_{x^{\prime}+1}-a_{\mathbf{k}^{\prime}+1}}, \alpha_{i_{1}}, \cdots, \alpha_{i_{s}}\right\}-\left\{\alpha_{i_{0}}, \beta_{a_{x^{\prime}+1}}, \cdots, \beta_{a_{k^{\prime}+1}}\right\}=\left\{\alpha_{j^{\prime}}, \alpha_{j^{\prime}}, \cdots, \alpha_{j^{\prime}}\right\},
$$

we get the desired conclusions $I \neq J$ and $A_{I}=A_{J}$.

\section{§4. The proof of the main lemma.}

4.1. This section is devoted to the proof of Lemma 3.6. Let $A=$ $\left(\alpha_{1}, \cdots, \alpha_{q}\right)(1 \leqq s<q \leqq 2 s)$ be a $q$-tuple of elements in $G$ with the property $\left(P_{q, s}\right)$ and $\alpha_{i}=1$ for some $i$. We note here we may assume $\alpha_{i_{0}}=1$ for an arbitrarily preassigned $i_{0}$. Indeed, we may study a new $q$-tuple $A^{\prime}:=\left(\alpha_{1} \alpha_{i_{0}}^{-1}, \cdots, \alpha_{q} \alpha_{i_{0}}^{-1}\right)$ instead of the original $A$. For, by the assumption, $\left\{\left\{\alpha_{1}, \cdots, \alpha_{q}\right\}\right\}=\left\{\left\{\alpha_{1} \alpha_{i_{0}}^{-1}, \cdots, \alpha_{q} \alpha_{i_{0}}^{-1}\right\}\right\}$ and so $t\left(A^{\prime}\right)=t(A)$.

Lemma 3.6 will be proved by the induction on $s$. For the case $s=1$, we have necessarily $q=2$ and $\alpha_{1}=\alpha_{2}(=1)$, which gives the desired conclusion. Consider next the case $s=2$. Then $q=3$ or $q=4$ and, after suitable changes of indices, we may assume $\alpha_{1}=\alpha_{2}=1$ by Lemma 3.5 and the above remark. If $q=3$, taking a combination $I=$ $((1,2)) \in \widetilde{\Im}_{3,2}$, we choose some $((i, j)) \in \widetilde{\Im}_{3,2}$ with $((i, j)) \neq((1,2))$ and $\alpha_{i} \alpha_{j}=$ $\alpha_{1} \alpha_{2}=1$. Then, necessarily, $\alpha_{i}=1$ or $\alpha_{j}=1$. In any case, $\alpha_{1}=\alpha_{2}=\alpha_{3}$ $=1$, whence $t\left(\alpha_{1}, \alpha_{2}, \alpha_{3}\right)=0$. For the case $s=2$ and $q=4$, we choose again a combination $((i, j))$ with $((i, j)) \neq((1,2))$ and $\alpha_{i} \alpha_{j}=\alpha_{1} \alpha_{2}$. If $\alpha_{i}=1$ or $\alpha_{j}=1$, we may write

$$
\alpha_{1}: \alpha_{2}: \alpha_{3}: \alpha_{4}=1: 1: 1: \beta
$$

with some $\beta \in G$ by a suitable change of indices. And, if $\alpha_{i} \neq 1$ and $\alpha_{j} \neq 1$, it may be written

$$
\alpha_{1}: \alpha_{2}: \alpha_{3}: \alpha_{4}=1: 1: \beta: \beta^{-1},
$$

where $\beta \neq 1$. In any case, $t\left(\alpha_{1}, \cdots, \alpha_{4}\right) \leqq 1$ and, if $t\left(\alpha_{1}, \cdots, \alpha_{4}\right)=1,\left(\alpha_{1}\right.$, $\left.\cdots, \alpha_{4}\right)$ is of the type (B).

In the following, we assume $s \geqq 3$ and Lemma 3.6 is valid if $s$ is replaced by a number smaller than $s$. And, we consider the case $t:=$ 
$t(\mathrm{~A}) \geqq s-1$ only, because, if otherwise, we have nothing to prove. Let $M_{0}:=\left\{i ; \alpha_{i}=1\right\}$ and $m_{0}:=\# M_{0}$, where $\# M$ denotes the number of elements in a set $M$. Since $A$ may be replaced by $\left\{\alpha_{1} \alpha_{i_{0}}^{-1}, \cdots, \alpha_{q} \alpha_{i_{0}}^{-1}\right\}$ for any $i_{0}$, we may assume $m_{0} \geqq \sharp\left\{i ; \alpha_{i}=\alpha_{j}\right\}$ for any $j(1 \leqq j \leqq q)$. Then, $m_{0} \geqq 2$ by Lemma 3.5. Now, we take an adequate base $\beta_{1}, \cdots, \beta_{t}$ of $\left\{\left\{\alpha_{1}, \cdots, \alpha_{q}\right\}\right\}$ as in (3.2) and express each $\alpha_{i}$ as (3.1) with integers $\ell_{i \tau}$. The proof of Lemma 3.6 are given separately for each of the following two cases;

Case $\alpha$. For each $\tau(1 \leqq \tau \leqq t), \ell_{1 \tau}, \cdots, \ell_{q_{\tau}}$ are all non-negative or all non-positive.

Case $\beta$. For some $\tau$, there exist distinct indices $i, j$ with $\ell_{i \tau}>0$ and $\ell_{j_{\tau}}<0$.

4.2. The proof of Lemma 3.6 for the case $\alpha$. For each $\tau$, after a replacement of $\beta_{\tau}$ by $\beta_{\tau}^{-1}$ if necessary, it may be assumed that $\ell_{i_{\tau}} \geqq 0$ for any $i$. Put

$$
M_{\tau}:=\left\{i ; \ell_{i \tau} \neq 0, \ell_{i_{\tau+1}}=\cdots=\ell_{i t}=0\right\}
$$

and $m_{\tau}:=\# M_{\tau}$ for each $\tau(1 \leqq \tau \leqq t)$.

We shall show first the following fact.

(4.1) For any subset $\left\{\tau_{1}, \cdots, \tau_{u}\right\}$ of the set $\{1,2, \cdots, t\}$ of indices, $m_{\tau_{1}}+$ $m_{\tau_{2}}+\cdots+m_{\tau_{u}} \neq s$.

Proof. Assume that $m_{\tau_{1}}+\cdots+m_{\tau_{u}}=s$ for some $\tau_{1}, \cdots, \tau_{u}$ and put

$$
M^{*}:=M_{\tau_{1}} \cup M_{\tau_{2}} \cup \cdots \cup M_{\tau_{u}}=\left\{i_{1}, i_{2}, \cdots, i_{s}\right\},
$$

where $1 \leqq \tau_{1}<\cdots<\tau_{u} \leqq t$ and $1 \leqq i_{1}<i_{2}<\ldots<i_{s} \leqq q$. By the assumption, there exists some $J=\left(\left(j_{1}, \cdots, j_{s}\right)\right) \in \widetilde{S}_{q, s}$ such that $I \neq J$ and

$$
\alpha_{i_{1}} \alpha_{i_{2}} \cdots \alpha_{i_{s}}=\alpha_{j_{1}} \alpha_{j_{2}} \cdots \alpha_{j_{s}} .
$$

If $M_{t} \cap M^{*}=\phi$, by expressing the both sides of (4.2) with $\beta_{1}, \cdots, \beta_{t}$ and observing the exponents of $\beta_{t}$ we see

$$
\sum_{\tau=1}^{s} \ell_{j_{\tau} t}=\sum_{\tau=1}^{s} \ell_{i_{\tau} t}=0
$$

whence $\ell_{j_{\tau} t}=0 \quad(1 \leqq \tau \leqq s)$ because $\ell_{i t} \geqq 0$ for any $i$. So, $M_{t} \cap\left\{j_{1}\right.$, $\left.\cdots, j_{s}\right\}=\phi$. And, if $M_{t} \cap M^{*} \neq \phi$, then $M_{t} \subset M^{*}$. In this case, 


$$
\sum_{\tau=1}^{s} \ell_{j_{\tau} t}=\sum_{\tau=1}^{s} \ell_{i_{\tau} t}=\sum_{i \in M_{t}} \ell_{i t},
$$

whence $M_{t} \subset\left\{j_{1}, j_{2}, \cdots, j_{t}\right\}$. In any case, we have

$$
M_{t} \cap\left\{i_{1}, \cdots, i_{s}\right\}=M_{t} \cap\left\{j_{1}, \cdots, j_{s}\right\} .
$$

Cancel $\alpha_{i}$ with $i \in M_{t}$ in the both sides of (4.2) and observe the exponents of $\beta_{t-1}$ of the obtained relation. Then, we can conclude that, if $M_{t-1} \cap M^{*}=\phi$,

$$
M_{t-1} \cap\left\{i_{1}, \cdots, i_{s}\right\}=M_{t-1} \cap\left\{j_{1}, \cdots, j_{s}\right\}=\phi
$$

and, if $M_{t-1} \cap M^{*} \neq \phi$,

$$
M_{t-1} \subset\left\{i_{1}, \cdots, i_{s}\right\} \cap\left\{j_{1}, \cdots, j_{s}\right\} .
$$

Therefore,

$$
\left(M_{t-1} \cup M_{t}\right) \cap\left\{i_{1}, \cdots, i_{s}\right\}=\left(M_{t-1} \cup M_{t}\right) \cap\left\{j_{1}, \cdots, j_{s}\right\} .
$$

Repeating this process, we get finally

$$
\left(M_{0} \cup M_{1} \cup \cdots \cup M_{t}\right) \cup\left\{i_{1}, \cdots, i_{s}\right\}=\left(M_{0} \cup \cdots \cup M_{t}\right) \cap\left\{j_{1}, \cdots, j_{s}\right\} .
$$

This contradicts the assumption $I \neq J$. Thus, we have the conclusion (4.1).

We shall prove next

(4.3) Under the above assumption, we have always $t \leqq s-1$. And, if $t=s-1$, then $q=2 s$ and one of the following two cases occurs;

(a) $m_{0}=s-1, m_{1}=m_{2}=\cdots=m_{s-1}=1$,

(b) $m_{0}=m_{1}=\cdots=m_{s-1}=2$.

Proof. We define the number $\sigma_{1}, \cdots, \sigma_{t}$ so that

$$
m_{\sigma_{1}} \geqq m_{\sigma_{2}} \geqq \cdots \geqq m_{\sigma_{t}} \text {. }
$$

Since $m_{0} \geqq 2$ and $m_{\sigma} \geqq 1$ for any $\sigma$,

$$
\begin{aligned}
2 s & \geqq q=m_{0}+\left(m_{\sigma_{1}}+\cdots+m_{\sigma_{t}}\right) \\
& \geqq 2+m_{\sigma_{1}}+(t-1) \\
& \geqq m_{\sigma_{1}}+s
\end{aligned}
$$

and so $m_{\sigma_{1}} \leqq s$. Take the largest number $u_{0}$ such that

$$
m^{*}:=m_{\sigma_{1}}+m_{\sigma_{2}}+\cdots+m_{\sigma_{u_{0}}} \leqq s .
$$


By (4.1), $m^{*}<s$. Assume $u_{0}=t$. Then,

$$
s-1 \leqq t \leqq m_{\sigma_{1}}+m_{\sigma_{2}}+\cdots+m_{\sigma_{t}}<s .
$$

So, $t=s-1, m_{o_{1}}=\cdots=m_{o_{t}}=1$ and $m_{0}=q-\left(m_{1}+\cdots+m_{t}\right)=q-$ $s+1$. If $q=2 s, m_{0}=s+1$ and so the case (a) of (4.3) occurs. For the case $q \leqq 2 s-1$, we have $m_{0} \leqq s$. We may put

$$
\alpha_{1}: \alpha_{2}: \cdots: \alpha_{q}=1: 1: \cdots: 1: \beta_{1}: \cdots: \beta_{s-1},
$$

where $\left\{\beta_{1}, \cdots, \beta_{s-1}\right\}$ is a basis of $\left\{\left\{\alpha_{1}, \cdots, \alpha_{q}\right\}\right\}$ and 1 is repeated at most $s$ times. For a combination $I=((1,2, \cdots, s))$, it is easily seen that there is no combination $J \in \mathfrak{\Im}_{q, s}$ with $I \neq J$ and $A_{I}=A_{J}$. The case $u_{0}=t$ and $q \leqq 2 s-1$ does not occur.

Now, let us consider the case $u_{0}<t$. Then, $m^{*}+m_{\sigma_{u_{0}+1}}>s$ and $m_{\sigma_{0}+1} \geqq 2$. Let $v:=\sharp\left\{\tau: m_{\tau}=1\right\}$. By (4.1), $m^{*}+v=m^{*}+m_{\sigma_{t-v+1}}+$ $\cdots+m_{\sigma_{t}}<s$. So,

$$
v \leqq s-m^{*}-1 \leqq\left(m^{*}+m_{\sigma_{0}+1}-1\right)-m^{*}-1=m_{\sigma_{0}+1}-2 \leqq m_{\sigma_{1}}-2 \text {. }
$$

On the other hand, since $m_{\sigma_{2}} \geqq \cdots \geqq m_{\sigma_{t-v}} \geqq 2$,

$$
\begin{aligned}
2 s & \geqq q=m_{0}+m_{\sigma_{1}}+\left(m_{\sigma_{2}}+\cdots+m_{\sigma_{t-v}}\right)+\left(m_{\sigma_{t-v+1}}+\cdots+m_{\sigma_{t}}\right) \\
& \geqq 2+m_{\sigma_{1}}+2(t-v-1)+v \\
& \geqq m_{\sigma_{1}}-v+2 t .
\end{aligned}
$$

Thus, we conclude $t \leqq s-1$. Let $t=s-1$. Then,

$$
m_{\sigma_{1}} \leqq v+2 s-2(s-1)=v+2 \leqq m_{\sigma_{u_{0}+1}} \leqq m_{\sigma_{1}} .
$$

We have necessarily $\tilde{m}:=m_{\sigma_{1}}=\cdots=m_{\sigma_{u_{0}+1}}=v+2$. Moreover, we can show $\tilde{m}=m_{\sigma_{\tau}}$ for any $\tau$ with $\tau \leqq t-v$. In fact, if $m_{\sigma_{\tau}}<\tilde{m}$ for some $\tau$ with $\tau \leqq t-v$, putting $v^{\prime}:=s-m^{*}-m_{\sigma_{\tau}}$, we see $0 \leqq v^{\prime} \leqq v$ and

$$
m^{*}+m_{\sigma_{\tau}}+m_{\sigma_{t-v^{\prime}+1}}+\cdots+m_{\sigma_{t}}=s,
$$

which contradicts (4.1). From these facts, it follows that

$$
\begin{aligned}
2 s & \geqq q=m_{0}+\left(m_{\sigma_{1}}+\cdots+m_{\sigma_{t-v}}\right)+\left(m_{\sigma_{t-v+1}}+\cdots+m_{\sigma_{t}}\right) \\
& \geqq 2+\tilde{m}(t-\tilde{m}+2)+\tilde{m}-2 \\
& =\tilde{m}(s-\tilde{m}+2)
\end{aligned}
$$

and so $\tilde{m}^{2}-(s+2) \tilde{m}+2 s \geqq 0$. Then, $\tilde{m} \geqq s$ or $\tilde{m} \leqq 2$. We know $\tilde{m} \leqq s$ and the case $\tilde{m}=s$ contradicts the assumption (4.1). Therefore $\tilde{m}=2$. 
This implies that $v=0$ and $m_{1}=m_{2}=\cdots=m_{t}=2$. In this case, since

$$
(2 \leqq) m_{0}=q-\left(m_{1}+\cdots+m_{t}\right) \leqq 2 s-(2 s-2)=2,
$$

the case (b) of (4.3) occurs. The proof of (4.3) is completed.

We go back to the proof of Lemma 3.6 for the case $(\alpha)$. The conclusion (i) of Lemma 3.6 was already shown in (4.3). We shall prove (ii) under the assumption $t=s-1$.

If the case (a) of (4.3) occurs, $q=2 s$ and we may write

$$
\alpha_{1}: \alpha_{2}: \cdots: \alpha_{2 s}=1: 1: \cdots: 1: \beta_{1}: \cdots: \beta_{s-1},
$$

where $\left\{\beta_{1}, \cdots, \beta_{s-1}\right\}$ is a basis of $\left\{\left\{\alpha_{1}, \cdots, \alpha_{2 s}\right\}\right\}$ and 1 is repeated $s+1$ times in the right-hand side. This is a special case of the type (B) of Lemma 3.6.

We assume now the case (b) of (4.3) occurs. Then, changing indices, we may put

$$
M_{0}:=\{1,2\}, \quad M_{1}:=\{3,4\}, \cdots, \quad M_{s-1}=\{2 s-1,2 s\}
$$

and

$$
\alpha_{1}=\alpha_{2}=1, \quad \alpha_{2 \tau+1}=\beta_{\tau}^{\ell_{\tau}}, \quad \alpha_{2 \tau+2}=\beta_{1}^{\ell_{\tau} 1} \beta_{2}^{\ell_{\tau} 2} \cdots \beta_{\tau}^{\ell_{\tau \tau}},
$$

where $1 \leqq \tau \leqq s-1$ and $\ell_{\tau}, \ell_{\sigma \tau}$ are integers with $\ell_{\tau}>0, \ell_{\tau \tau}>0, \ell_{\sigma \tau} \geqq 0$ for any $\sigma, \tau$. Here, we can show that

$$
A^{*}:=\left(\alpha_{1}, \alpha_{2}, \cdots, \alpha_{2 s-4}\right)
$$

satisfies the condition $\left(P_{2 s-4, s-2}\right)$. In fact, for any given combination $I^{*}=\left(\left(i_{1}, \cdots, i_{s-2}\right)\right)$ of elements in $\{1,2, \cdots, 2 s-4\}$, if we take a combination $J:=\left(\left(j_{1}, \cdots, j_{s}\right)\right) \in \widetilde{\mathfrak{S}}_{2 s, s}$ with $J \neq I:=\left(\left(i_{1}, \cdots, i_{s-2}, 2 s-1,2 s\right)\right)$ and $A_{I}=A_{J}$, we see easily

$$
1 \leqq j_{1}<\ldots<j_{s-2} \leqq 2 s-4<j_{s-1}=2 s-1<j_{s}=2 s
$$

by observing the exponents of $\beta_{s-1}$ and $\beta_{s-2}$ in the expression of the both sides of the relation $A_{I}=A_{J}$ with $\beta_{\tau}(1 \leqq \tau \leqq s-1)$. Therefore, $J^{*}:=$ $\left(\left(j_{1}, \cdots, j_{s-2}\right)\right) \in \widetilde{J}_{2 s-4, s-2}$ satisfies the conditions $I^{*} \neq J^{*}$ and $A^{*}{ }_{J^{*}}=A^{*}{ }_{I^{*}}$ By the induction hypothesis, $A^{*}=\left(\alpha_{1}, \cdots, \alpha_{2 s-4}\right)$ is of the type (A) or (B). But, there is no possibility of the type (B), because $\ell_{i_{\tau}} \geqq 0$ for any $i, \tau$ and $\# M_{\sigma}=2(0 \leqq \sigma \leqq s-1)$. So, $A^{*}$ is of the type (A), namely, $s$ is odd and $\alpha_{2 \tau+1}=\alpha_{2 \tau+2}$ if $1 \leqq \tau \leqq s-3$. Now, for a combination $I:=$ 
$((3,4, \cdots, 2 r+1,2 r+2,2 s-2,2 s-1,2 s)) \in \widetilde{\mathfrak{J}}_{2 s, s}$ take some $J=\left(\left(j_{1}, \cdots j_{s}\right)\right)$ with $I \neq J$ and $A_{I}=A_{J}$ according to the assumption, where $r=\frac{s-3}{2}$. By expressing $A_{I}=A_{J}$ with $\beta_{1}, \cdots, \beta_{s-1}$ and observing the exponents of $\beta_{s-1}$, we have necessarily $j_{s-3} \leqq 2 s-4, j_{s-1}=2 s-1, j_{s}=2 s$ and $j_{s-2}=$ $2 s-3$ or $=2 s-2$. If $j_{s-2}=2 s-2$, then there is a non-trivial algebraic relation among $\beta_{1}, \cdots, \beta_{s-2}$, which is a contradiction. So, $j_{s-2}=2 s-3$. Moreover, if we observe the exponents of $\beta_{1}, \cdots, \beta_{s-3}$, it is easily seen that $j_{1}=3, j_{2}=4, \cdots, j_{s-3}=2 r+2$. The relation $A_{I}=A_{J}$ implies $\alpha_{2 s-2}=\alpha_{2 s-3}$. For $I^{\prime}:=((1,2, \cdots, 2 r+1,2 r+2,2 s))$ taking a combination $J^{\prime}$ with $J^{\prime} \neq I^{\prime}$ and $A_{I^{\prime}}=A_{J^{\prime}}$, we can show also $\alpha_{2 s-1}=\alpha_{2 s}$ in the same manner as the above. Therefore, $A$ is of the type (A), which completes the proof of Lemma 3.6 for the case $\alpha$.

4.3. The proof of Lemma 3.6 for the case $\beta$. Changing indices, for the exponents $\ell_{i t}$ of $\beta_{t}$ in the expression (3.1) of $\alpha_{i}(1 \leqq i \leqq q)$ we may assume that

$$
\ell_{1 t} \geqq \cdots \geqq \ell_{n_{++1}}=\cdots=\ell_{n_{+}+n_{0} t}=0>\ell_{n_{+} n_{0}+1 t} \geqq \cdots \geqq \ell_{q t},
$$

where $n_{+} \geqq 1$ and $n_{-}:=q-\left(n_{+}+n_{0}\right) \geqq 1$ by the assumption. Moreover, after a replacement of $\beta_{t}$ by $\beta_{t}^{-1}$ if necessary, we may assume $n_{+} \leqq n_{-}$.

We shall show first

$$
\text { Under the above assumptions, } 1 \leqq s-n_{+}<n_{0} \leqq 2\left(s-n_{+}\right) \text {and }
$$
$A^{*}=\left(\alpha_{n_{+}+1}, \cdots, \alpha_{n_{+}+n_{0}}\right)$ has the property $\left(P_{n_{0}, s-n_{+}}\right)$.

Proof. Since $\left\{\beta_{1}, \cdots, \beta_{t}\right\}$ is an adequate basis, $\alpha_{i_{\tau}}=\beta_{\tau}^{\ell_{r}}\left(\ell_{\tau} \neq 0\right)$ for suitable $i_{1}, \cdots, i_{t}$, whence $\ell_{i_{\tau}}=0$ for $\tau=1,2, \cdots, t-1$. Therefore,

$$
n_{0} \geqq m_{0}+(t-1) \geqq 2+(t-1) \geqq s \text {. }
$$

We have then

$$
n_{0}>s-n_{+}>s-\left(n_{+}+n_{-}\right)=s-\left(q-n_{0}\right) \geqq n_{0}-s \geqq 0 .
$$

And, since $n_{+} \leqq n_{-}$,

$$
2\left(s-n_{+}\right) \geqq 2 s-\left(n_{+}+n_{-}\right) \geqq q-\left(q-n_{0}\right)=n_{0} .
$$

Now, let us take an arbitrary combination $I^{*}:=\left(\left(i_{n_{+}+1}, \cdots, i_{s}\right)\right)$ of elements in $\left\{n_{+}+1, \cdots, n_{+}+n_{0}\right\}$. By the assumption of $A=\left(\alpha_{1}, \cdots, \alpha_{q}\right)$, 
for a combination $I:=\left(\left(1,2, \cdots, n_{+}, i_{n_{+}+1}, \cdots, i_{s}\right)\right)$ there is some $J=\left(\left(j_{1}\right.\right.$, $\left.\left.\cdots, j_{s}\right)\right) \in \mathfrak{\Im}_{q, s}$ with $J \neq I$ and $A_{I}=A_{J}$. Observe the exponents of $\beta_{t}$ of $A_{I}$ and $A_{J}$. As is easily seen,

$$
j_{1}=1, \cdots, j_{n_{+}}=n_{+}, \quad n_{+}+1 \leqq j_{n_{+}+1}<\ldots<j_{s} \leqq n_{+}+n_{0} .
$$

This concludes $A_{I^{*}}=A_{J^{*}}$ for a combination $J^{*}:=\left(\left(j_{n_{+}+1}, \cdots, j_{s}\right)\right)\left(\neq I^{*}\right)$. The assertion (4.4) is proved.

Obviously, the system $\left\{\beta_{1}, \cdots, \beta_{t-1}\right\}$ is a basis of $\left\{\left\{\alpha_{n_{+}+1}, \cdots, \alpha_{n_{+}+n_{0}}\right\}\right\}$. We can conclude from the induction hypothesis

$$
t-1 \leqq s-n_{+}-1 \leqq s-2
$$

and so $t \leqq s-1$. This completes the proof of (i) of Lemma 3.6. Let $t=s-1$. Then, by the above inequalities, $n_{+}=1$ and $A^{*}=\left(\alpha_{n_{+}+1}\right.$, $\left.\cdots, \alpha_{n_{+}+n_{0}}\right)$ is of the type (A) or of the type (B). In any case, $n_{0}=$ $2\left(s-n_{+}\right)=2 s-2$ and

$$
n_{-}=q-\left(n_{0}+n_{+}\right) \leqq 2 s-(2 s-2+1)=1,
$$

whence $n_{-}=1$ and $q=2 s$. In this situation, we shall show

(4.5) $A^{*}$ cannot be of the type $(A)$.

Proof. Let $A^{*}$ be of the type (A). Then, we may put

$$
\alpha_{1}: \cdots: \alpha_{2 s}=1: 1: \beta_{1}^{\ell_{1}}: \beta_{1}^{\ell_{1}}: \cdots: \beta_{s-2}^{\ell_{s-2}}: \beta_{s-2}^{\ell_{s-2}}: \beta_{s-1}^{\ell_{s-1}}: \beta_{1}^{\ell_{1}^{1}} \cdots \beta_{s-1}^{\ell_{s}^{\prime}-1}
$$

by a suitable change of indices, where $s-1$ is odd and $\ell_{\sigma}, \ell_{\tau}^{\prime}$ are integers with $\ell_{\sigma}>0(1 \leqq \sigma \leqq s-1)$ and $\ell_{s-1}^{\prime}<0$. Consider first the case that some $\ell_{\tau}^{\prime}$ with $1 \leqq \tau \leqq s-2$, say $\ell_{1}^{\prime}$, is positive. Putting $r=s / 2$, for $I:=((3,4, \cdots, 2 r-1,2 r, 2 s-1,2 s)) \in \widetilde{S}_{2 s, s}$ we take $J=\left(\left(j_{1}, \cdots, j_{s}\right)\right) \in \mathfrak{\Im}_{2 s, s}$ such that $J \neq I$ and $A_{I}=A_{J}$. By comparing the exponents of $\beta_{1}$ of $A_{I}$ and $A_{J}$, we see easily $j_{s}=2 s$. And, by observing the exponents of $\beta_{s-1}$ of them, we have also $j_{s-1}=2 s-1$. Then, since $I \neq J$, we get a nontrivial relation among $\beta_{1}, \cdots, \beta_{s-1}$, which is impossible. Consider next the case $\ell_{\tau}^{\prime} \leqq 0$ for any $\tau$. Take in this case a combination $J^{\prime} \in \mathfrak{I}_{2 s, s}$ such that $J^{\prime} \neq I^{\prime}$ and $A_{J^{\prime}}=A_{I^{\prime}}$ for $I^{\prime}:=((1,2, \cdots, 2 r-1,2 r)) \in \widetilde{\mathcal{S}}_{2 s, s}$. By comparing the exponents of $\beta_{1}, \cdots, \beta_{s-1}$ of the both sides of $A_{J^{\prime}}=A_{I^{\prime}}$, we have necessarily a non-trivial relation among $\beta_{1}, \cdots, \beta_{s-1}$. This is a contradiction. Thus, (4.5) holds.

To complete the proof, it suffices to show 
(4.6) In the case $A^{*}$ is of the type $(B),\left(\alpha_{1}, \cdots, \alpha_{2 s}\right)$ is also of the type (B).

Proof. Changing indices, we assume $A^{*}=\left(\alpha_{1}, \cdots, \alpha_{2 s-2}\right)$. We may put by the assumption

$$
\begin{aligned}
& \alpha_{1}: \alpha_{2}: \cdots: \alpha_{2 s} \\
& \quad=1: \cdots: 1: \beta_{1}^{\prime}: \cdots: \beta_{s-2}^{\prime}:\left(\beta_{1}^{\prime} \cdots \beta_{a_{1}}^{\prime}\right)^{-1}: \cdots:\left(\beta_{a_{k-2}+1}^{\prime} \cdots \beta_{a_{k-1}}^{\prime}\right)^{-1}: \alpha_{2 s-1}: \alpha_{2 s}
\end{aligned}
$$

and $\beta_{\tau}^{\prime}=\beta_{\tau}^{\ell_{\tau}}(1 \leqq \tau \leqq s-2), \alpha_{2 s-1}=\beta_{s-1}^{\ell_{s}-1}, \alpha_{2 s}=\beta_{1}^{\ell_{1}^{\prime}} \beta_{2}^{\ell_{2}^{\prime}} \cdots \beta_{s-1}^{\ell_{s}^{\prime}-1}$ for a basis $\left\{\beta_{1}, \cdots, \beta_{s-1}\right\}$ of $\left\{\left\{\alpha_{1}, \cdots, \alpha_{2 s}\right\}\right\}$, where 1 appears $s-k+1$ times repeatedly and $1 \leqq k \leqq s-1, a_{k}-a_{k-1} \leqq s-k$ and $\ell_{1}, \cdots, \ell_{s-1}, \ell_{1}^{\prime}, \cdots, \ell_{s-1}^{\prime}$ are integers with $\ell_{\tau}>0, \ell_{s-1}^{\prime}<0$. Then, $\ell_{\tau}^{\prime} \geqq 0$ if $1 \leqq \tau \leqq a_{k-1}$. In fact, for example, if $\ell_{1}^{\prime}<0$, we have a non-trivial relation among $\beta_{1}, \cdots, \beta_{s-1}$ by observing a combination $J \in \mathfrak{S}_{2 s, s}$ with $J \neq I, A_{J}=A_{I}$ for $I:=((s-k+3$, $\cdots, 2 s-k, 2 s-1,2 s))$. Now, for $I^{\prime}:=((s-k+2, \cdots, 2 s-k-1,2 s-$ $1,2 s)$ ) let us take a combination $J^{\prime}:=\left(\left(j_{1}, \cdots, j_{s}\right)\right)$ with $J^{\prime} \neq I^{\prime}, A_{J^{\prime}}=A_{I^{\prime}}$. If $\ell_{\tau}^{\prime}>0$ for some $\tau(1 \leqq \tau \leqq s-2)$, then we have easily $j_{s}=2 s$ and a non-trivial relation among $\beta_{1}, \cdots, \beta_{s-1}$. Therefore, $\ell_{\tau}^{\prime} \leqq 0$ for any $\tau(1 \leqq$ $\tau \leqq s-1)$ and, particularly, $\ell_{\tau}^{\prime}=0$ if $1 \leqq \tau \leqq a_{k-1}$. Moreover, as is easily seen, none of $\alpha_{j_{\tau}}(1 \leqq \tau \leqq s)$ are equal to $\alpha_{2 s-k}, \cdots, \alpha_{2 s-2}, \alpha_{2 s}$. If we cancel out some of $\alpha_{s-k+2}, \cdots, \alpha_{2 s-k-1}, \alpha_{2 s-1}$ in the both sides of the relation $A_{I^{\prime}}=A_{J^{\prime}}$, we obtain

$$
\beta_{\tau_{1}}^{\ell_{1}} \cdots \beta_{\tau_{b}-1}^{\ell_{\tau_{b}-1}} \alpha_{2 s}=\alpha_{\sigma_{1}} \alpha_{\sigma_{2}} \cdots \alpha_{\sigma_{b}}=1
$$

where $1 \leqq b \leqq s-k+1, \quad a_{k-1}<\tau_{1}<\ldots<\tau_{b-1} \leqq s-1$ and $1 \leqq \sigma_{1}<$ $\ldots<\sigma_{b} \leqq s-k+1$. Changing notations and indices suitably, we may put

$$
\alpha_{2 s}=\left(\beta_{a_{k-1}+1}^{a_{a_{k-1}+1}} \cdots \beta_{a_{k}}^{a_{k}}\right)^{-1} .
$$

If we replace each $\beta_{\tau}^{\ell_{\tau}}$ by $\beta_{\tau}$, we get the conclusion that $A$ is of the type (B). We have thus Lemma 3.6.

\section{§5. The smallest algebraic set including the image of $\boldsymbol{f} \times \boldsymbol{g}$.}

5.1. Let $f, g$ be meromorphic maps of $C^{n}$ into $P^{N}(C)$. Assume that, for $2 N+2$ hyperplanes $H_{1}, \cdots, H_{2 N+2}$ in $P^{N}(C)$ located in general position, $f\left(C^{n}\right) \varnothing H_{i}, g\left(C^{n}\right) \varnothing H_{i}$ and $\nu\left(f, H_{i}\right)=\nu\left(g, H_{i}\right)(1 \leqq i \leqq 2 N+2)$.

DEFINITION 5.1. We define the set $V_{f, g}$ to be the smallest algebraic 
set in $P^{N}(C) \times P^{N}(C)$ which contains points $(f \times g)(z)=(f(z), g(z))$ for any $z \in C^{n}-(I(f) \cup I(g))$, where $I(f)$ and $I(g)$ are sets defined as (2.1) for the maps $f$ and $g$.

$V_{f, g}$ is an irreducible algebraic set.

Indeed, if $V_{f, g}=V_{1} \cup V_{2}$ for two algebraic sets $V_{1}, V_{2}$ with $V_{i} \subsetneq V_{f, g}$ then $A_{i}:=(f \times g)^{-1}\left(V_{i}\right)(i=1,2)$ are analytic sets in $C^{n}$ and $C^{n}=A_{1} \cup$ $A_{2}$. Since $C^{n}$ is irreducible, $C^{n}=A_{1}$ or $C^{n}=A_{2}$. Therefore, $V_{f, q}=V_{1}$ or $V_{f, g}=V_{2}$, which contradicts the assumption.

As in $\S 2$, taking admissible representations of $f$ and $g$, we define holomorphic functions $F_{f}^{H_{i}}, F_{g}^{H_{i}}$ by (2.2) for each $H_{i}(1 \leqq i \leqq 2 N+2)$ and $h_{i}=F_{f}^{H_{i}} / F_{g}^{H_{i}}$, where at least one $h_{i}$ is assumed to be constant by a suitable choice of admissible representations.

We shall prove now the following theorem.

THEOREM 5.3. Suppose that among the functions $h_{1}, \cdots, h_{2 N+2}$ there exist $2 s$ functions $h_{i_{1}}, \cdots, h_{i_{2 s}}$ such that the canonical images $\alpha_{1}:=\left[h_{i_{1}}\right]$, $\cdots, \alpha_{2 s}:=\left[h_{i_{2 s}}\right]$ of $h_{i}$ into the factor group $H^{*} / C^{*}$ do not satisfy the condition $\left(P_{2 s, s}\right)$. Then, for the number $t=t\left(\left[h_{1}\right], \cdots,\left[h_{2 N+2}\right]\right)$

$$
\operatorname{dim} V_{f, g} \leqq N-s+t .
$$

Before the proof of Theorem 5.3, we shall give

COROLLARY 5.4. (i) $V_{f, g}$ is always of dimension $\leqq N$.

(ii) If $\operatorname{dim} V_{f, g}=N$, the system $\left(\left[h_{1}\right], \cdots,\left[h_{2 N+2}\right]\right)$ in $H^{*} / C^{*}$ has the property $\left(P_{2 t+2, t+1}\right)$ for the number $t=t\left(\left[h_{1}\right], \cdots,\left[h_{2 N+2}\right]\right)$.

Proof of Corollary 5.4. We choose $h_{i_{1}}, \cdots, h_{i_{2 t}}$ among $h_{1}, \cdots, h_{2 N+2}$ suitably such that $t=t\left(\left[h_{i_{1}}\right], \cdots,\left[h_{i_{2 t}}\right]\right)$. Then, $\left(\left[h_{i_{1}}\right], \cdots,\left[h_{i_{2 t}}\right]\right)$ do not satisfy the condition $\left(P_{2 t, t}\right)$. For, if not, $t\left(\left[h_{i_{1}}\right], \cdots,\left[h_{i_{2 t}}\right]\right) \leqq t-1$ by Lemma 3.6, (i). Putting $s=t$, we can apply Theorem 5.3. So, under the assumption that Theorem 5.3 is valid, we obtain

$$
\operatorname{dim} V_{f, g} \leqq(N-s)+s=N .
$$

On the other hand, if some $(2 t+2)$-tuple $\left(\left[h_{i_{1}}\right], \cdots,\left[h_{i_{2 t+2}}\right]\right)\left(1 \leqq i_{1}<\ldots<\right.$ $\left.i_{2 t+2} \leqq 2 N+2\right)$ do not satisfy the condition $\left(P_{2 t+2, t+1}\right)$, we can conclude

$$
\operatorname{dim} V_{f, g} \leqq N-(t+1)+t=N-1
$$

from Theorem 5.3, which shows the conclusion (ii) of Corollary 5.4. 
5.2. The proof of Theorem 5.3. Suppose that for $2 s$ functions of $h_{1}$, $\cdots, h_{2 N+2}$, say $h_{1}, \cdots, h_{s}, h_{N+2}, \cdots, h_{N+s+1},\left(\left[h_{1}\right], \cdots,\left[h_{s}\right],\left[h_{N+2}\right], \cdots,\left[h_{N+s+1}\right]\right)$ do not satisfy the condition $\left(P_{2 s, s}\right)$. Since functions $h_{i}$ are not changed by a change of homogeneous coordinates on $P^{N}(C)$ the hyperplanes $H_{i}$ may be written as

$$
H_{i}: a_{i}^{1} w_{1}+\cdots+a_{i}^{N+1} w_{N+1}=0 \quad(1 \leqq i \leqq 2 N+2)
$$

such that $a_{i}^{j}=\delta_{i}^{j}(1 \leqq i, j \leqq N+1)$, where $\delta_{i}^{j}=0$ if $i \neq j$ and $=1$ if $i=j$. Then, any minor of a matrix $\left(\alpha_{N+j+1}^{i} ; 1 \leqq i, j \leqq N+1\right)$ does not vanish. Let us take functions $\eta_{1}, \cdots, \eta_{t} \in \boldsymbol{H}^{*}$ such that $\left\{\left[\eta_{1}\right], \cdots,\left[\eta_{t}\right]\right\}$ gives a basis for $\left\{\left\{\left[h_{1}\right], \cdots,\left[h_{2 N+2}\right]\right\}\right\}$ in $\boldsymbol{H}^{*} / C^{*}$. Then each $h_{i}(1 \leqq i \leqq 2 N+2)$ can be written uniquely as

$$
h_{i}=c_{i} \eta_{1}^{\ell_{i 1}} \eta_{2}^{\ell_{i 2}} \cdots \eta_{t}^{\ell_{i t}} \quad\left(c_{i} \in C^{*}, \ell_{i_{\tau}} \in Z\right)
$$

and $\eta_{1}^{\ell_{1}} \eta_{2}^{\ell_{2}} \cdots \eta_{t}^{\ell_{t}} \notin C^{*}$ for any $\ell_{\tau} \in Z$ with $\left(\ell_{1}, \ell_{2}, \cdots, \ell_{t}\right) \neq(0,0, \cdots, 0)$. Put $\ell_{i t+1}=-\left(\ell_{i 1}+\cdots+\ell_{i t}\right)$ and define rational functions

$$
H_{i}(u)=c_{i} u_{1}^{\ell_{1} 1} u_{2}^{\ell_{2}} \cdots u_{t+1}^{\ell_{i t+1}} \quad(1 \leqq i \leqq 2 N+2)
$$

of $t+1$ variables $u=\left(u_{1}, \cdots, u_{t+1}\right)$. Each $H_{i}(u)$ is written as $H_{i}(u)=$ $H_{i}^{+}(u) / H_{i}^{-}(u)$ with homogeneous polynomials $H_{i}^{+}(u)=c_{i} \prod_{t=1}^{t+1} u^{\ell_{i \tau}^{+}}$and $H_{i}^{-}(u)=\prod_{\tau=1}^{t+1} u_{\tau}^{i_{\tau}}$ of the same degree, where $\ell_{i \tau}^{+}=\max \left(\ell_{i_{\tau}}, 0\right), \ell_{i_{\tau}}^{-}=$ $-\min \left(\ell_{i_{\tau}}, 0\right)$. Now, we consider the space $X:=P^{t}(C) \times P^{N}(C) \times P^{N}(C)$ and an algebraic set $V^{*}$ consisting of all points

$$
(u, v, w)=\left(u_{1}: \cdots: u_{t+1}, v_{1}: \cdots: v_{N+1}, w_{1}: \cdots: w_{N+1}\right) \in X
$$

satisfying the equations

$$
\left(\sum_{j=1}^{N+1} a_{i}^{j} v_{j}\right) H_{i}^{-}(u)=c_{0}\left(\sum_{j=1}^{N+1} a_{i}^{j} w_{j}\right) H_{i}^{+}(u)
$$

$(1 \leqq i \leqq 2 N+2)$ for some non-zero constant $c_{0}$. Let $\pi_{i}(i=1,2,3)$ be the canonical projections defined as $\pi_{1}(u, v, w)=u, \pi_{2}(u, v, w)=v$ and $\pi_{3}(u, v, w)=w\left((u, v, w) \in V^{*}\right)$. We define an algebraic set $V^{* *}$ as the union of all irreducible components $V_{t}^{*}$ of $V^{*}$ satisfying the conditions

$$
\begin{aligned}
& \text { (1) } \pi_{1}\left(V_{\imath}^{*}\right)=P^{t}(C), \\
& \text { (2) } \pi_{2}\left(V_{\imath}^{*}\right) \varnothing \bigcup_{i=1}^{2 N+2} H_{i} \text { and } \pi_{3}\left(V_{\imath}^{*}\right) \varnothing \bigcup_{i=1}^{2 N+2} H_{i} .
\end{aligned}
$$

And, we put $\tilde{V}:=\left(\pi_{2} \times \pi_{3}\right)\left(V^{* *}\right)$, which is a subvariety of $P^{N}(C)$. Then, 


$$
V_{f, g} \subset \tilde{V}
$$

To see this, we recall the definition of $h_{i}$ and the relation (5.5). For admissible representations $f=f_{1}: \cdots: f_{N+1}$ and $g=g_{1}: \cdots: g_{N+1}$, it holds that

$$
\sum_{j=1}^{N+1} a_{i}^{j} f_{j}=\left(\sum_{j=1}^{N+1} a_{i}^{j} g_{j}\right) H_{i}\left(\eta_{1}, \cdots, \eta_{t}, \eta_{t+1}\right) \quad(1 \leqq i \leqq 2 N+2),
$$

where $\eta_{t+1} \equiv 1$. This shows that, for a holomorphic map $\eta=\eta_{1}: \eta_{2}$ : $\cdots: \eta_{t+1}$ of $C^{n}$ into $P^{t}(C)$,

$$
(\eta \times f \times g)(z):=(\eta(z), f(z), g(z)) \in V^{*} \quad\left(z \in C^{n}-(I(f) \cup I(g))\right) .
$$

Then, by the same argument as in the proof of (5.2) we see easily $(\eta \times f \times g)\left(C^{n}\right) \subset V_{\iota_{0}}^{*}$ for an irreducible component $V_{\iota_{0}}^{*}$ of $V^{*}$. On the other hand, by the assumption, $f\left(C^{n}\right) \subset \pi_{1}\left(V_{c_{0}}^{*}\right), g\left(C^{n}\right) \subset \pi_{2}\left(V_{c_{0}}^{*}\right), f\left(C^{n}\right) \varnothing$ $\bigcup_{i=1}^{2 N+2} H_{i}$ and $g\left(C^{n}\right) \varnothing \bigcup_{i=1}^{2 N+2} H_{i}$. Therefore, $V_{i 0}^{*}$ satisfies the condition (2) of (5.7). Moreover, by the property of the functions $\eta_{\tau}$ and the conclusion (2.9), $\eta\left(C^{n}\right)$ does not included in any proper subvariety of $P^{t}(C)$. So, $\eta\left(C^{n}\right) \subset \pi_{1}\left(V_{t_{0}}^{*}\right)$ implies $\pi_{1}\left(V_{t_{0}}^{*}\right)=P^{t}(C)$. By definition, $V_{\iota_{0}}^{*} \subset V^{* *}$. And, we see

$$
(f \times g)\left(C^{n}\right) \subset\left(\pi_{2} \times \pi_{3}\right)\left(V^{* *}\right)=\tilde{V} .
$$

We have thus (5.8) by the definition of $V_{f, g}$.

Now, consider the equations

$$
\begin{aligned}
\sum_{j=1}^{s} a_{i}^{j}\left(H_{i}(u)-H_{j}(u)\right) w_{j}=-\sum_{j=s+1}^{N+1} a_{i}^{j}\left(H_{i}(u)-\right. & \left.H_{j}(u)\right) w_{j} \\
& (N+2 \leqq j \leqq N+s+1)
\end{aligned}
$$

obtained by substitutions of $v_{i}=c_{0} H_{i}(u) w_{i}(1 \leqq i \leqq N+1)$ into the relations $(5.6)_{i}$ for $i=N+2, \cdots, N+s+1$. We can prove here the following fact, which will be shown later.

$$
\Psi(u):=\operatorname{det}\left(a_{N+i+1}^{j}\left(H_{N+i+1}(u)-H_{j}(u)\right) ; 1 \leqq i, j \leqq s\right) \not \equiv 0 .
$$

By virtue of (5.10), the equations (5.9) can be resolved as

$$
w_{\tau}=\Phi_{\tau}\left(u_{1}, \cdots, u_{t+1}, w_{s+1}, \cdots, w_{N+1}\right) \quad(1 \leqq \tau \leqq s)
$$

with rational functions $\Phi_{\tau}$, whose denominators $\chi_{\tau}$ may be chosen as functions of $u_{1}, \cdots ; u_{t+1}$ only. This implies that for any point $(u, v, w)$ $=\left(u_{1}: \cdots: u_{t+1}, v_{1}: \cdots: v_{N+1}, w_{1}: \cdots: w_{N+1}\right)$ in $V^{* *} w_{1}, \cdots, w_{s}$ are uniquely 
determined by the values $u_{1}, \cdots, u_{t+1}, w_{s+1}, \cdots, w_{N+1}$ if $\chi_{\tau}(u) \neq 0(1 \leqq \tau \leqq s)$. On the other hand, each $v_{j}(1 \leqq j \leqq N+1)$ is determined by $u_{1}, \cdots, u_{t+1}$, $w_{1}, \cdots, w_{N+1}$ in view of $(5.6)_{i}$ for $i=1,2, \cdots, N+1$ if $u_{1} u_{2} \cdots u_{t+1} \neq 0$. From these facts, we can conclude the map $\pi^{*}$ of $V^{* *}$ into $C^{t} \times C^{N-s}$ defined as

$$
\begin{array}{r}
\pi^{*}\left(u_{1}: \cdots: u_{t+1}, v_{1}: \cdots: v_{N+1}, w_{1}: \cdots: w_{N+1}\right) \\
\quad=\left(\left(\frac{u_{1}}{u_{t+1}}, \cdots, \frac{u_{t}}{u_{t+1}}\right),\left(\frac{w_{s+1}}{w_{N+1}}, \cdots, \frac{w_{N}}{w_{N+1}}\right)\right)
\end{array}
$$

is injective if the definition domain is restricted to the range

$$
\begin{aligned}
& u_{1} u_{2} \cdots u_{t+1} \neq 0, \quad v_{1} v_{2} \cdots v_{N+1} \neq 0, \quad w_{1} w_{2} \cdots w_{N+1} \neq 0, \\
& \chi_{\tau}(u) \neq 0 \quad(1 \leqq \tau \leqq s) .
\end{aligned}
$$

By definition, any irreducible component of $V^{* *}$ intersects with the range (5.11) in $X$. It follows

$$
\operatorname{dim} V_{f, g} \leqq \operatorname{dim} \tilde{V} \leqq \operatorname{dim} V^{* *} \leqq t+(N-s)
$$

Because, in general, in the case there exists a holomorphic map $f$ of an irreducible complex space $X_{1}$ into $X_{2}$, we can conclude $\operatorname{dim} X_{1} \leqq \operatorname{dim} X_{2}$ if $f$ is injective on some non-empty open set, and $\operatorname{dim} X_{2} \leqq \operatorname{dim} X_{1}$ if $f$ is surjective.

To complete the proof of Theorem 5.3, it remains to prove the assertion (5.10). To this end, we rewrite $\Psi(u)$ as

$$
\Psi(u)=\operatorname{det}\left(\begin{array}{cc}
I_{s}, & I_{s}^{\prime} \\
A, & A^{\prime}
\end{array}\right),
$$

where $I_{s}$ is the unit matrix of order $s$ and $A=\left(\alpha_{j}^{N+i+1} ; 1 \leqq i, j \leqq s\right)$, $I_{s}^{\prime}=\left(\delta_{i}^{j} H_{i}(u) ; 1 \leqq i, j \leqq s\right)$ and $A^{\prime}=\left(a_{N+i+1}^{j} H_{N+i+1}(u) ; 1 \leqq i, j \leqq s\right)$. Then, we see

$$
\Psi(\eta):=\Psi\left(\eta_{1}, \cdots, \eta_{t}, 1\right)=\operatorname{det}\left(\begin{array}{cc}
I_{s} & I_{s}^{\prime \prime} \\
A & A^{\prime \prime}
\end{array}\right),
$$

where $I_{s}^{\prime \prime}=\left(\delta_{i}^{j} h_{i} ; 1 \leqq i, j \leqq s\right)$ and $A^{\prime \prime}=\left(a_{N+i+1}^{j} h_{N+i+1} ; 1 \leqq i, j \leqq s\right)$. On the other hand, it is easily seen that any minor of a $2 s \times s$ matrix $\left(\begin{array}{l}I_{s} \\ A\end{array}\right)$ of order $s$ does not vanish. If $\Psi(\eta) \equiv 0$, then $\left(\left[h_{1}\right], \cdots,\left[h_{s}\right],\left[h_{N+2}\right]\right.$, $\left.\cdots,\left[h_{N+s+1}\right]\right)$ satisfies the condition $\left(P_{2 s, s}\right)$ by $(2.8)$, which contradicts the 
assumption. Therefore, $\Psi(\eta) \not \equiv 0$. We can conclude the assertion (5.10).

\section{§6. Algebraically non-degenerate meromorphic maps.}

6.1. We give first

DEFINITION 6.1. Let $f$ be a meromorphic map of $C^{n}$ into $P^{N}(C)$. We shall call $f$ to be algebraically non-degenerate if $f\left(C^{n}\right)$ is not included in any proper subvariety of $P^{N}(C)$.

As in the previous sections, consider meromorphic maps $f, g$ of $C^{n}$ into $P^{N}(C)$ such that for hyperplanes $H_{1}, \cdots, H_{2 N+2}$ in general position $f\left(C^{n}\right) \varnothing H_{i}, g\left(C^{n}\right) \varnothing H_{i}$ and $\nu\left(f, H_{i}\right)=\nu\left(g, H_{i}\right)(1 \leqq i \leqq 2 N+2)$.

(6.2) If $f$ or $g$ is algebraically non-degenerate, then the algebraic set $V_{f, g}$ defined as in Definition 5.1 is of dimension $N$.

Proof. It may be assumed that $f$ is algebraically non-degenerate. Obviously, $f\left(C^{n}\right) \subset \pi_{1}\left(V_{f, g}\right)$. By the assumption, $\pi_{1}\left(V_{f, g}\right)$ cannot be a proper subvariety of $P^{N}(C)$. Therefore

$$
\operatorname{dim} V_{f, g} \geqq \operatorname{dim} \pi_{1}\left(V_{f, g}\right)=N \text {. }
$$

Corollary 5.4 yields $\operatorname{dim} V_{f, g}=N$.

q.e.d.

Let $h_{i}(1 \leqq i \leqq 2 N+2)$ be functions defined as (2.4) and assume that at least one of them is constant.

Proposition 6.3. In the above situation, if $f$ or $g$ is algebraically non-degenerate, there exist elements $\beta_{1}, \cdots, \beta_{t}$ in $H^{*} / C^{*}$ such that

$$
\begin{aligned}
& {\left[h_{1}\right]:\left[h_{2}\right]: \cdots:\left[h_{2 N+2}\right]} \\
& \quad=1: 1: \cdots: 1: \beta_{1}: \cdots: \beta_{t}:\left(\beta_{1} \cdots \beta_{a_{1}}\right)^{-1}: \cdots:\left(\beta_{a_{k-1}+1} \cdots \beta_{t}\right)^{-1},
\end{aligned}
$$

where 1 appears $2 N-k-t+2$ times repeatedly in the right hand side and $t=t\left(\left[h_{1}\right], \cdots,\left[h_{2 N+2}\right]\right), a_{x}-a_{x-1} \leqq t-k+1$ (let $a_{0}=0$ and $\left.a_{k}=t\right)$.

To prove this, we need the following

LEMMA 6.5. Assume that $h_{i}(1 \leqq i \leqq 2 N+2)$ are represented as

$$
h_{i}=c_{i} \eta_{1}^{\ell_{i 1}} \eta_{2}^{\ell_{i 2}} \cdots \eta_{t}^{\ell_{i t}} \quad\left(c_{i} \in C^{*}, \ell_{i \tau} \in Z\right)
$$

with functions $\eta_{1}, \cdots, \eta_{t} \in H^{*}$, where $t=t\left(\left[h_{1}\right], \cdots,\left[h_{2 N+2}\right]\right)$. Then, there 
is no possibility that, for some $\tau$, exactly one of integers $\ell_{1 \tau}, \ell_{2 \tau}, \cdots, \ell_{2 N+2 \tau}$ is not zero and the others vanish.

Proof. Without loss of generality, we may assume

$$
\ell_{1 t}=\ell_{2 t}=\cdots=\ell_{2 N+1 t}=0, \quad \ell_{2 N+2 t}=1 .
$$

As is stated in $\S 2$, there is a relation (2.5) among $h_{1}, \cdots, h_{2 N+2}$. Therefore

$$
\operatorname{det}\left(a_{i}^{1}, \cdots, a_{i}^{N+1}, a_{i}^{1} H_{i}(\eta), \cdots, a_{i}^{N+1} H_{i}(\eta) ; 1 \leqq i \leqq 2 N+2\right) \equiv 0,
$$

where $H_{i}(\eta)$ are given by substitutions of $u_{\tau}=\eta_{\tau}$ into

$$
H_{i}(u)=c_{i} u_{1}^{\ell_{i}} u_{2}^{\ell_{i}} \cdots u_{t}^{\ell_{i t}} .
$$

According to (2.9), we have then

$$
\operatorname{det}\left(a_{i}^{1}, \cdots, a_{i}^{N+1}, a_{i}^{1} H_{i}(u), \cdots, a_{i}^{N+1} H_{i}(u) ; 1 \leqq i \leqq 2 N+2\right) \equiv 0
$$

as a rational function of $u_{1}, \cdots, u_{t}$. Substitute $u_{t}=0$ into this identity. We get by the assumption

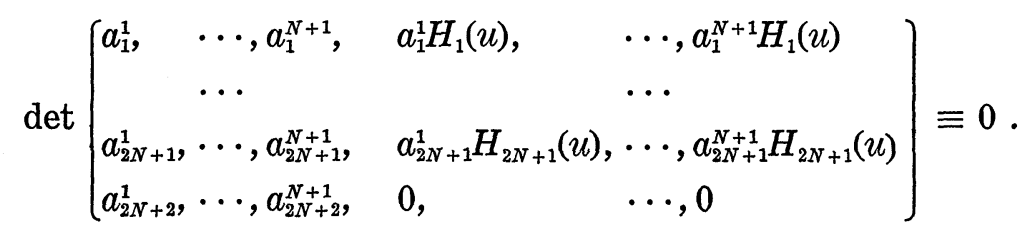

It then follows

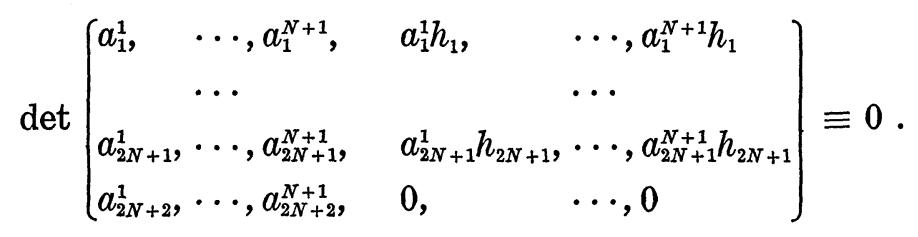

In this situation, by the well-known argument any solutions $\left(x_{1}, \cdots, x_{N+1}\right.$, $\left.y_{1}, \cdots, y_{N+1}\right)$ of the linear equations

$$
\sum_{j=1}^{N+1} a_{i}^{j} x_{j}=\sum_{j=1}^{N+1} a_{i}^{j} h_{i}(z) y_{j} \quad(1 \leqq i \leqq 2 N+1)
$$

satisfy simultaneously an equation

$$
\sum_{j=1}^{N+1} a_{2 N+2}^{j} x_{j}=0
$$

for any fixed $z$. In particularly, the identities 


$$
\sum_{j=1}^{N+1} a_{i}^{j} f_{j}(z)=\sum_{j=1}^{N+1} a_{i}^{j} h_{i}(z) g_{j}(z) \quad(1 \leqq i \leqq 2 N+1)
$$

yield

$$
\sum_{j=1}^{N+1} a_{2 N+2}^{j} f_{j} \equiv 0
$$

This shows $f\left(C^{n}\right) \subset H_{2 N+2}$, which contradicts the assumption. We have thus Lemma 6.5.

q.e.d.

6.2. Proof of Proposition 6.3. By the assumption and (6.2), $\operatorname{dim} V_{f, g}$ $=N$ and, by virtue of Corollary 5.4, (ii), the system $\left(\left[h_{1}\right], \cdots,\left[h_{2 N+2}\right]\right)$ satisfies the condition $\left(P_{2 t+2, t+1}\right)$. In Lemma 3.4 considering the case $q=2 N+2, r=2 t+2$ and $s=t+1$, we can conclude that $2 N-2 t+2$ elements of $\left[h_{1}\right], \cdots,\left[h_{2 N+2}\right]$ are equal to each others. By suitable choices of an admissible representation of $f$ and indices, we may assume

$$
h_{1} \sim h_{2} \sim h_{2 t+3} \sim \cdots \sim h_{2 N+2} \sim 1 \text {. }
$$

Then, $A:=\left(\left[h_{1}\right], \cdots,\left[h_{2 t+2}\right]\right)$ satisfies the condition $\left(P_{2 t+2, t+1}\right)$ and $t=t(A)$. According to Lemma 3.6, $\left(\left[h_{1}\right], \cdots,\left[h_{2 t+2}\right]\right)$ is represented as one of the types (A) and (B) of Lemma 3.6, (ii) if we put $s=t+1$ and $\alpha_{i}=\left[h_{i}\right]$. For the case of the type (B), we may put by a suitable change of indices

$$
\begin{aligned}
& {\left[h_{1}\right]:\left[h_{2}\right]: \cdots:\left[h_{2 N+2}\right]} \\
& \quad=1: 1: \cdots: 1: \beta_{1}: \cdots: \beta_{t}:\left(\beta_{1} \cdots \beta_{a_{1}}\right)^{-1}: \cdots:\left(\beta_{a_{k-1}+1} \cdots \beta_{a_{k}}\right)^{-1},
\end{aligned}
$$

where 1 appears $2 N+2-(t+k)$ times and $a_{k}-a_{x-1} \leqq t+1-k$. Moreover, by Lemma 6.5 there is no possibility $a_{k}<t$. We have the conclusion of Proposition 6.3.

Let us consider the case $A$ is of the type (A). We may put then

$$
\left[h_{1}\right]:\left[h_{2}\right]: \cdots:\left[h_{2 N+2}\right]=1: 1: \beta_{1}: \beta_{1}: \cdots: \beta_{t}: \beta_{t}: 1: \cdots: 1
$$

with suitable $\beta_{1}, \cdots, \beta_{t}$ in $H^{*} / C^{*}$, where $t$ is an even number. We shall show here $t=N$. Suppose $t<N$. As was already seen, any chosen $2 t+2$ elements among $\left[h_{1}\right], \cdots,\left[h_{2 N+2}\right]$, particularly, $\alpha_{1}:=\left[h_{1}\right], \cdots, \alpha_{2 t+1}$ : $=\left[h_{2 t+1}\right], \alpha_{2 t+2}:=\left[h_{2 t+3}\right]$ satisfies the condition $\left(P_{2 t+2, t+1}\right)$. For a combination $I=((1,2, \cdots, t, 2 t+2)) \in \widetilde{\Im}_{2 t+2, t+1}$ observe $J=\left(\left(j_{1}, \cdots, j_{t+1}\right)\right) \in \widetilde{\Im}_{2 t+2, t+1}$ such that $J \neq I$ and

$$
\alpha_{i_{1}} \alpha_{i_{2}} \cdots \alpha_{i_{t+1}}=\alpha_{j_{1}} \alpha_{j_{2}} \cdots \alpha_{j_{t+1}}
$$


Then, we have necessarily a relation among $\beta_{1}, \cdots, \beta_{t}$ because $t$ is even. This is a contradition. Thus, $t=N$.

To complete the proof of Proposition 6.3, we shall prove that (6.6) cannot occur for $t=N$. Assume the contrary. Changing indices, we may put $h_{N+1} \equiv 1$ and $h_{N+i+1}=c_{i} h_{i}(1 \leqq i \leqq N+1)$ for some constants $c_{i} \in C^{*}$, where $\left[h_{1}\right], \cdots,\left[h_{N}\right]$ give a basis of $\left\{\left\{\left[h_{1}\right], \cdots,\left[h_{2 N+2}\right]\right\}\right\}$. Moreover, for these choices of indices, given hyperplanes

$$
H_{i}: a_{i}^{1} w_{1}+a_{i}^{2} w_{2}+\cdots+a_{i}^{N+1} w_{N+1}=0 \quad(1 \leqq i \leqq 2 N+2)
$$

may be assumed to satisfy the condition that $a_{i}^{j}=\delta_{i}^{j}(1 \leqq i, j \leqq N+1)$. Then, by substituting $f_{i}=h_{i} g_{i}(1 \leqq i \leqq N+1)$ into the identities

$$
\sum_{j=1}^{N+1} a_{N+i+1}^{j} f_{j}=c_{i} h_{i}\left(\sum_{j=1}^{N+1} a_{N+i+1}^{j} g_{j}\right) \quad(1 \leqq i \leqq N+2),
$$

we have relations

$$
\alpha_{i}^{1} h_{1}+\alpha_{i}^{2} h_{2}+\cdots+\alpha_{i}^{N} h_{N}+\alpha_{i}^{N+1}=0 \quad(1 \leqq i \leqq N+1),
$$

where

$$
\alpha_{i}^{j}:=a_{N+i+1}^{j} g_{j}-c_{i} \delta_{i}^{j}\left(\sum_{j=1}^{N+1} a_{N+i+1}^{j} g_{j}\right) .
$$

Eliminate $h_{1}, \cdots, h_{N}$ from these equations. We obtain

$$
\chi\left(g_{1}, \cdots, g_{N+1}\right):=\operatorname{det}\left(\alpha_{i}^{j} ; 1 \leqq i, j \leqq N+1\right) \equiv 0 .
$$

By the assumption, we may consider $g$ to be algebraically non-degenerate. So, there is no non-trivial algebraic relation among $g_{1}, \ldots, g_{N+1}$. This implies that $\chi$ vanishes identically as a polynomial of independent variables $g_{1}, \cdots, g_{N+1}$. In particular, for any $i$, if we put $g_{i}=1, g_{1}=\cdots=$ $g_{i-1}=g_{i+1}=\cdots=g_{N+1}=0$,

$$
\begin{aligned}
& \chi(0, \cdots, 0,1,0, \cdots, 0) \\
& \quad=(-1)^{N} c_{1} \cdots c_{i-1}\left(1-c_{i}\right) c_{i+1} \cdots c_{N+1} a_{N+2}^{i} \cdots a_{2 N+2}^{i}=0 .
\end{aligned}
$$

Therefore, $c_{1}=c_{2}=\cdots=c_{N+1}=1$, because $a_{j}^{i} \neq 0$ by the assumption that $H_{1}, \cdots, H_{2 N+2}$ are located in general position. Since

$$
\operatorname{det}\left(\alpha_{i}^{j} ; 1 \leqq i, j \leqq N\right) \not \equiv 0
$$

by the algebraically non-degeneracy of $g$, we can solve the functions $h_{i}$ from $N$ equations $(6.7)_{i}(1 \leqq i \leqq N)$ by the well-known Cramer's formula. 
For example, we get $h_{1} \equiv 1$. This contradicts the fact that $\left(\left[h_{1}\right], \ldots,\left[h_{N}\right]\right)$ is a basis of $\left\{\left\{\left[h_{1}\right], \cdots,\left[h_{2 N+2}\right]\right\}\right\}$. We have thus the desired conclusion. Proposition 6.3 is completely proved.

Remark 6.8. We cannot assert that all cases of the conclusion of Proposition 6.3 occur. In fact, for example, in the case $N=3$, the only case $t=3, k=3, a_{1}=a_{2}=a_{3}=1$ is possible (cf., $\S 7.2$ ).

Proposition 6.3 can be restated in a form not including the functions $h_{i}$ explicitly. In the same situation as in Proposition 6.3, we consider holomorphic functions $F_{f}^{H_{i}}=\sum_{j=1}^{N+1} a_{i}^{j} f_{j}$ and $F_{g}^{H_{i}}=\sum_{j=1}^{N+1} a_{i}^{j} g_{j}(1 \leqq i \leqq$ $2 N+2)$ defined as (2.2), where

$$
H_{i}: a_{i}^{1} w_{1}+\cdots+a_{i}^{N+1} w_{N+1}=0
$$

and $f, g$ have admissible representations $f=f_{1}: f_{2}: \cdots: f_{N+1}, g=g_{1}: g_{2}$ : $\cdots: g_{N+1}$ respectively.

THEOREM 6.9. If either $f$ or $g$ is algebraically non-degenerate, there are relations between $f$ and $g$ such that, after a suitable change of indices,

$$
\begin{aligned}
& F_{f}^{H_{1}}=c_{1} F_{g}^{H_{1}}, \cdots, F_{f}^{H_{\ell}}=c_{\ell} F_{g}^{H_{\ell}}
\end{aligned}
$$

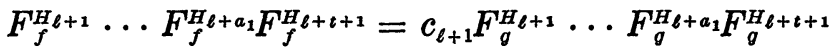

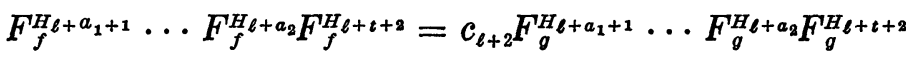

$$
\begin{aligned}
& F_{f}^{H_{\ell+a_{k-1}+1}} \cdots F_{f}^{H_{\ell+t}} F_{f}^{H_{2 N+2}}=c_{\ell+k} F_{g}^{H_{\ell+a_{k-1}+1}} \cdots F_{g}^{H_{\ell+t}} F_{g}^{H_{2 N+2}} \text {, }
\end{aligned}
$$

where $c_{i} \in C^{*}, 0 \leqq t \leqq N, 2 \leqq \ell \leqq N+1, k=2 N-\ell-t+2, a_{k}-a_{k-1} \leqq$ $t-k+1$ (put $\left.a_{0}=0, a_{k}=t\right)$.

The proof is evident by Proposition 6.3 except the assertion $\ell \leqq$ $N+1$. This is due to the fact that, if $\ell \geqq N+2, f$ is (linearly) degenerate as was shown in the proof of Theorem II in [3], p. 12.

6.3. Now, we give the uniqueness theorem of meromorphic maps stated in $\S 1$.

THEOREM 6.10. Let $f, g$ be meromorphic maps of $C^{n}$ into $P^{N}(C)$ such that $f\left(C^{n}\right) \varnothing H_{i}, \quad g\left(C^{n}\right) \varnothing H_{i}$ and $\nu\left(f, H_{i}\right)=\nu\left(g, H_{i}\right)$ for $2 N+3$ hyperplanes $H_{i}$ in general position. If $f$ or $g$ is algebraically non-degenerate, then $f \equiv g$. 
Proof. Assume that $f \not \equiv g$ and consider the functions $h_{1}, \cdots, h_{2 N+3}$ defined as (2.4). By (2.8) and Lemma 3.4, there are at least three mutually distinct indices, say 1,2,3, such that $h_{1} \sim h_{2} \sim h_{3}$. Apply Proposition 6.3 to maps $f, g$ and $2 N+2$ hyperplanes $H_{2}, \cdots, H_{2 N+3}$. After a suitable change of indices, we may put

$$
\begin{aligned}
& {\left[h_{2}\right]: \cdots:\left[h_{2 N+3}\right]} \\
& \quad=1: 1: \cdots: 1: \beta_{1}: \cdots: \beta_{t}:\left(\beta_{1} \cdots \beta_{a_{1}}\right)^{-1}: \cdots:\left(\beta_{a_{k-1+1}} \cdots \beta_{t}\right)^{-1},
\end{aligned}
$$

where $\beta_{1}, \cdots, \beta_{t} \in \boldsymbol{H}^{*} / \boldsymbol{C}^{*}, t=t\left(\left[h_{1}\right], \cdots,\left[h_{2 N+3}\right]\right)(\geqq 1), 1 \leqq a_{1}<\cdots<a_{k-1}$ $<t$ and 1 is repeated $2 N+2-t-k$ times. Then, if we take functions $\eta_{i}$ with $\left[\eta_{i}\right]=\beta_{i}(1 \leqq i \leqq t)$ and represent functions $h_{i}(1 \leqq i \leqq 2 N+2)$ as

$$
h_{i}=c_{i} \eta_{1}^{\ell_{i 1}} \cdots \eta_{t}^{\ell_{i t}} \quad\left(c_{i} \in C^{*}, \ell_{i j} \in Z\right),
$$

$\ell_{2 N+2-k t}=1$ and $\ell_{i t}=0$ for any other $i$ because $h_{2 N+3}$ is omitted. This contradicts Lemma 6.5. Thus, we can conlcude $f \equiv g$. q.e.d.

In Theorem 6.3, the number $2 N+3$ of given hyperplanes cannot be replaced by $2 N+2$. In fact, we can construct two distinct algebraically non-degenerate moromorphic maps $f$ and $g$ of $C^{n}$ into $P^{N}(C)$ such that $\nu\left(f, H_{i}\right)=\nu\left(g, H_{i}\right)$ for $2 N+2$ hyperplanes $H_{i}$ in general position. Put $N=2 M$ in the case $N$ is even and $N=2 M+1$ in the case $N$ is odd. Take $2 N+2$ hyperplanes $H_{1}, \cdots, H_{2 N+2}$ defined as (2.3) which are located in general position and satisfies the conditions;

(i) $a_{i}^{j}=\delta_{i}^{j} \quad(1 \leqq i, j \leqq N+1)$,

(ii) $a_{N+M+i+1}^{j}=a_{N+i+1}^{M+j}, a_{N+M+i+1}^{M+j}=a_{N+i+1}^{j} \quad(1 \leqq i, j \leqq M)$,

(iii) $a_{N+i+1}^{N+1}=a_{2 N+2}^{i}=1(1 \leqq i \leqq N+1)$ in the case $N$ is even and $a_{N+M+i+1}^{N}=a_{N+i+1}^{N+1}, a_{N+M+i+1}^{N+1}=a_{N+i+1}^{N}, a_{2 N+1}^{i}=a_{2 N+1}^{M+i}, a_{2 N+2}^{i}=-a_{2 N+2}^{M+i}(1 \leqq i \leqq$ $M), a_{2 N+1}^{N}=a_{2 N+1}^{N+1}, a_{2 N+2}^{N}=-a_{2 N+2}^{N+1}$ in the case $N$ is odd.

And, choosing algebraically independent functions $\eta_{1}, \cdots, \eta_{N}$ in $H^{*}$, we put

$$
\left(\eta_{1}^{*}, \eta_{2}^{*}, \cdots, \eta_{2 N+2}^{*}\right):=\left(\eta_{1}, \cdots, \eta_{M}, \eta_{1}^{-1}, \cdots, \eta_{M}^{-1}, 1, \eta_{M+1}, \cdots, \eta_{2 M}, \eta_{M+1}^{-1}, \cdots, \eta_{2 M}^{-1}, 1\right)
$$

in the case $N$ is even and

$$
\begin{aligned}
& \left(\eta_{1}^{*}, \eta_{2}^{*}, \cdots, \eta_{2 N+2}^{*}\right) \\
& \quad:=\left(\eta_{1}, \cdots, \eta_{M}, \eta_{1}^{-1}, \cdots, \eta_{M}^{-1}, \eta_{N}, \eta_{N}^{-1}, \eta_{M+1}, \cdots, \eta_{2 M}, \eta_{M+1}^{-1}, \cdots, \eta_{2 M}^{-1}, 1,-1\right)
\end{aligned}
$$

in the case $N$ is odd. We define meromorphic maps $f=f_{1}: f_{2}: \cdots: f_{N+1}$ 
and $g=g_{1}: g_{2}: \cdots: g_{N+1}$ of $C^{n}$ into $P^{N}(C)$ by the condition

$$
\sum_{i=1}^{N+1} \beta_{i}^{j} g_{j}=0 \quad(1 \leqq i \leqq N)
$$

and

$$
f_{i}=\eta_{i}^{*} g_{i} \quad 1 \leqq i \leqq N+1
$$

where

$$
\beta_{i}^{j}:=a_{N+i+1}^{j}\left(\eta_{N+i+1}^{*}-\eta_{j}^{*}\right)
$$

As is easily seen,

$$
\operatorname{det}\left(\beta_{i}^{j}\right) \equiv 0
$$

Therefore, in addition to (6.11), we have

$$
\sum_{j=1}^{N+1} a_{i}^{j} f_{j}=\eta_{i}^{*}\left(\sum_{j=1}^{N+1} a_{i}^{j} g_{j}\right) \quad(1 \leqq i \leqq 2 N+2)
$$

and so $f$ and $g$ satisfy the desired conditions $\nu\left(f, H_{i}\right)=\nu\left(g, H_{i}\right)$ $(1 \leqq i \leqq 2 N+2)$.

\section{§. Meromorphic maps into $P^{2}(C)$ or $P^{3}(C)$.}

7.1. In the last section of the previous paper [3], the author investigated the possible types of relations between two meromorphic maps $f$ and $g$ of $C^{n}$ into $P^{2}(C)$ satisfying the condition $\nu\left(f, H_{i}\right)=\nu\left(g, H_{i}\right)$ for six hyperplanes $H_{i}(1 \leqq i \leqq 6)$ in general position. In this place, we shall study them for the possible cases more precisely under the assumption that $f$ or $g$ is algebraically non-degenerate. In the following, we shall exclude the trivial case $f \equiv g$.

According to Proposition 6.3, the functions $h_{i}:=F_{f}^{H_{i}} / F_{g}^{H_{i}}(1 \leqq i \leqq 6)$ defined as (2.4) may be assumed to be written as (6.4) with some $\beta_{1}$, $\cdots, \beta_{t}$ in $H^{*} / C^{*}$ after a suitable change of indices, where $t=t\left(\left[h_{1}\right]\right.$, $\left.\cdots,\left[h_{6}\right]\right)$. Here, 1 appears at most three times by the assumption $f \neq \equiv$. So, $t=2$ and there are only two possible cases;
(a) $\left[h_{1}\right]: \cdots:\left[h_{6}\right]=1: 1: 1: \beta_{1}: \beta_{2}:\left(\beta_{1} \beta_{2}\right)^{-1}$,
( $\beta$ ) $\left[h_{1}\right]: \cdots:\left[h_{6}\right]=1: 1: \beta_{1}: \beta_{2}: \beta_{1}^{-1}: \beta_{2}^{-1}$. 
Let us study first the case $(\alpha){ }^{*}$ ) By suitable choices of homogeneous coordinates on $P^{2}(C)$ and admissible representations $f=f_{1}: f_{2}: f_{3}$ and $g=g_{1}: g_{2}: g_{3}$, we may put

$$
\begin{aligned}
& H_{i}: w_{i}=0 \quad(i=1,2,3) \\
& H_{4}: a w_{1}+b w_{2}+w_{3}=0 \\
& H_{5}: c w_{1}+d w_{2}+w_{3}=0 \\
& H_{6}: w_{1}+w_{2}+w_{3}=0
\end{aligned}
$$

and

$$
\begin{aligned}
& f_{1}=x_{1} g_{1}, f_{2}=x_{2} g_{2}, f_{3}=g_{3} \\
& F_{f}^{H_{4}}=\eta_{1} F_{g}^{H_{4}}, F_{f}^{H_{5}}=\eta_{2} F_{g}^{H_{4}}, F_{f}^{H_{6}}=x_{3}\left(\eta_{1} \eta_{2}\right)^{-1} F_{g}^{H_{6}},
\end{aligned}
$$

where $a, b, c, d, x_{1}, x_{2}, x_{3} \in C^{*}, \eta_{1}, \eta_{2} \in H^{*}$ with $t\left(\left[\eta_{1}\right],\left[\eta_{2}\right]\right)=2$ and $F_{f}^{H_{i}}, F_{g}^{H_{i}}$ are holomorphic functions defined as (2.2) for the above $H_{i}, f$ and $g$. We have then

$$
F_{f}^{H_{4}} F_{f}^{H_{5}} F_{f}^{H_{6}}=x_{3} F_{g}^{H_{4}} F_{g}^{H_{5}} F_{g}^{H_{6}} .
$$

Here, the left hand side can be rewritten with $g_{1}, g_{2}, g_{3}$. Since $g$ may be assumed to be algebraically non-degenerate, this is regarded as an identity of polynomials of independent variables $g_{1}, g_{2}, g_{3}$. By the uniqueness of factorization of a polynomial each factor in one side of this identity coincides with some factor in the other side. From this fact, we can conclude easily

$$
x_{1}=\omega, \quad x_{2}=\omega^{2}, \quad x_{3}=1
$$

and

$$
a=\omega, \quad b=\omega^{2}, \quad c=\omega^{2}, \quad d=\omega
$$

after a suitable change of indices, where $\omega$ denotes a primitive third root of unity. Then, by eliminating $f_{1}, f_{2}, f_{3}$ from the relations (7.2) and resolving $g_{1}, g_{2}, g_{3}$ we obtain

$$
g=g_{1}: g_{2}: g_{3}=1+\omega^{2} \eta_{1}+\omega \eta_{1} \eta_{2}: \omega^{2}+\eta_{1}+\omega \eta_{1} \eta_{2}: \omega\left(1+\eta_{1}+\eta_{1} \eta_{2}\right),
$$

*) In [3], pp. $21 \sim 22$, some statements should be corrected. By corrected calculations given in this paper the relation (7.4) in [3], p. 21 has a system of solutions with the desired properties as an equation with unknowns $c^{i}$ and $a_{j}^{i}$. The type

$$
\left(h_{1}, \cdots, h_{6}\right)=\left(1, c_{2}, c_{3}, h, h^{*}, c_{4}\left(h h^{*}\right)^{-1}\right)
$$

should be called to be of the type (VIII). 
which is algebraically non-degenerate. And, if we consider a transformation

$$
L_{1}: \quad w_{1}: w_{2}: w_{3} \mapsto \omega w_{1}: \omega^{2} w_{2}: w_{3}
$$

of $P^{2}(C), f$ and $g$ are related as $L_{1} \cdot g=f$. We note here that $L_{1}$ is a projective linear transformation of $P^{2}(C)$ onto itself which maps hyperplanes $H_{1}, H_{2}, \cdots, H_{6}$ onto $H_{1}, H_{2}, H_{3}, H_{5}, H_{6}, H_{4}$ respectively.

Let us consider next the case $(\beta)$. For the given hyperplanes (7.1) and the above functions $f_{i}, g_{i}, F_{f}^{H_{i}}$ and $F_{g}^{H_{i}}$, we may put

$$
\begin{aligned}
& f_{1}=\eta_{1} g_{1}, \quad f_{2}=\eta_{2} g_{2}, \quad f_{3}=g_{3} \\
& F_{f}^{H_{4}^{4}}=y_{1} \eta_{1}^{-1} g_{1}, \quad F_{f}^{H_{5}}=y_{2} \eta_{2}^{-1} F_{g}^{H_{5}}, \quad F_{f}^{H_{6}}=y_{3} F_{g}^{H_{6}}
\end{aligned}
$$

after a change of indices, where $y_{1}, y_{2}, y_{3} \in C^{*}, \eta_{1}, \eta_{2} \in H^{*}$ and $t\left(\left[\eta_{1}\right],\left[\eta_{2}\right]\right)$ $=2$. By eliminating $f_{i}, g_{i}$ from these relations, we get

$$
\left|\begin{array}{lll}
a\left(\eta_{1}^{2}-y_{1}\right) & b\left(\eta_{1} \eta_{2}-y_{1}\right) & \eta_{1}-y_{1} \\
c\left(\eta_{1} \eta_{2}-y_{2}\right) & d\left(\eta_{2}^{2}-y_{2}\right) & \eta_{2}-y_{2} \\
\eta_{1}-y_{3} & \eta_{2}-y_{3} & 1-y_{3}
\end{array}\right| \equiv 0,
$$

which may be regarded as an identity with independent variables $\eta_{1}, \eta_{2}$. By elementary calculations we see

$$
y_{1}=y_{2}=y_{3}=1, \quad b+c=2 a, \quad a=d .
$$

On the other hand, we have by (7.3)

$$
\begin{aligned}
& f_{3}=g_{3} \\
& f_{1}\left(a f_{1}+b f_{2}+f_{3}\right)=g_{1}\left(a g_{1}+b g_{2}+g_{3}\right) \\
& f_{2}\left(c f_{1}+d f_{2}+f_{3}\right)=g_{2}\left(c g_{1}+d g_{2}+g_{3}\right) \\
& f_{1}+f_{2}=g_{1}+g_{2},
\end{aligned}
$$

which implies $f_{1}=g_{1}$ or $f_{1}=\frac{a g_{1}+b g_{2}+g_{3}}{b-a}$. The former is the excluded case $f \equiv g$. For the latter case, we obtain

$$
g=g_{1}: g_{2}: g_{3}=1-\eta_{2}: \eta_{1}-1:(a-b) \eta_{1} \eta_{2}+a \eta_{1}-a \eta_{2}+b-a
$$

and maps $f$ and $g$ are related as $L_{2} \cdot g=f$ with a projective linear transformation

$$
L_{2}: \quad w_{1}: w_{2}: w_{3} \mapsto \frac{a w_{1}+b w_{2}+w_{3}}{b-a}: \frac{c w_{1}+d w_{2}+w_{3}}{c-d}: w_{3}
$$


of $P^{2}(C)$ which maps $H_{1}, H_{2}, \cdots, H_{6}$ onto $H_{4}, H_{5}, H_{3}, H_{1}, H_{2}, H_{6}$, respectively.

7.2. We shall study next algebraically non-degenerate meromorphic maps $f$ and $g$ of $C^{n}$ into $P^{3}(C)$ such that $f \neq \equiv g$ and $\nu\left(f, H_{i}\right)=\nu\left(g, H_{i}\right)$ for eight hyperplanes $H_{i}(1 \leqq i \leqq 8)$ in general position. For the functions $h_{i}(1 \leqq i \leqq 8)$ defined as (2.6), since we have only to consider the case $t=t\left(\left[h_{1}\right], \cdots,\left[h_{8}\right]\right) \leqq 4$, the possible cases of Proposition 6.3 are reduced to the following four types;

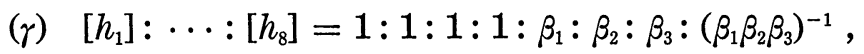

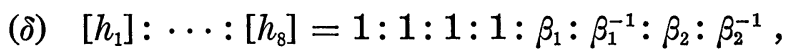

$$
\begin{aligned}
& \text { (ع) }\left[h_{1}\right]: \cdots:\left[h_{8}\right]=1: 1: 1: \beta_{1}: \beta_{2}:\left(\beta_{1} \beta_{2}\right)^{-1}: \beta_{3}: \beta_{3}^{-1} \text {, } \\
& \text { (ל) }\left[h_{1}\right]: \cdots:\left[h_{8}\right]=1: 1: \beta_{1}: \beta_{1}^{-1}: \beta_{2}: \beta_{2}^{-1}: \beta_{3}: \beta_{3}^{-1} \text {. }
\end{aligned}
$$

We can choose homogeneous coordinates on $P^{3}(C)$ so that

$$
\begin{aligned}
& H_{i}: w_{i}=0 \quad(i=1,2,3,4) \\
& H_{j+4}: a_{j}^{1} w_{1}+a_{j}^{2} w_{2}+a_{j}^{3} w_{3}+a_{j}^{4} w_{4}=0 \quad(j=1,2,3,4),
\end{aligned}
$$

where we may assume $a_{i}^{j}=1$ whenever $i=4$ or $j=4$.

For the case $(\gamma)$ or $(\delta)$, meromorphic maps $f=f_{1}: f_{2}: f_{3}: f_{4}$ and $g=g_{1}: g_{2}: g_{3}: g_{4}$ are related as

$$
f_{1}=x_{1} g_{1}, \quad f_{2}=x_{2} g_{2}, \quad f_{3}=x_{3} g_{3}, \quad f_{4}=g_{4}
$$

with some $x_{1}, x_{2}, x_{3} \in C^{*}$. Let us consider the functions $F_{f}^{H_{i}}$ and $F_{g}^{H_{i}}$ defined as (2.2). We obtain a relation

$$
F_{f}^{H_{5}} F_{f}^{H_{6}} F_{f}^{H_{7}} F_{f}^{H_{8}}=x_{A} F_{g}^{H_{5}} F_{g}^{H_{6}} F_{g}^{H_{7}} F_{g}^{H_{8}}
$$

in the case $(\gamma)$ and

$$
\begin{aligned}
& F_{f}^{H_{5}} F_{f}^{H_{6}}=x_{4}^{\prime} F_{g}^{H_{5}} F_{g}^{H_{6}}, \\
& F_{f}^{H_{7}} F_{f}^{H_{8}}=x_{5}^{\prime} F_{g}^{H_{7}} F_{g}^{H_{8}},
\end{aligned}
$$

in the case $(\delta)$, where $x_{4}, x_{4}^{\prime}, x_{5}^{\prime} \in C^{*}$. By (7.5), the left hand sides of these relations can be rewritten with $g_{1}, \cdots, g_{4}$ By the assumption, $g_{1}$, $\cdots, g_{4}$ may be considered as independent variables in the obtained relations. In both cases $(\gamma)$ and $(\delta)$, by comparing the factors of the both sides of these identities as in the consideration of the case $(\alpha)$, we can conclude that all possible choices of constants $a_{i}^{j}$ with the desired property contradict the assumption that any minor of the matrix $\left(a_{i}^{j}\right)$ does not vanish. The cases $(\gamma)$ and $(\delta)$ are both impossible. 
Next, we shall study the case $(\varepsilon)$. We may put then

$$
\begin{aligned}
& f_{1}=x_{1} g_{1}, \quad f_{2}=x_{2} g_{2}, \quad f_{3}=x_{3}\left(\eta_{1} \eta_{2}\right)^{-1} g_{3}, \quad f_{4}=x_{4} \eta_{3}^{-1} g_{4} \\
& \sum_{j=1}^{4} a_{i}^{j} f_{j}=\eta_{i}\left(\sum_{j=1}^{4} a_{i}^{j} g_{j}\right) \quad(i=1,2,3,4)
\end{aligned}
$$

after a change of indices, where $x_{1}, \cdots, x_{4} \in \boldsymbol{C}^{*}, \eta_{1}, \eta_{2}, \eta_{3} \in \boldsymbol{H}^{*}, t\left(\left[\eta_{1}\right],\left[\eta_{2}\right]\right.$, $\left.\left[\eta_{3}\right]\right)=3$ and, for convenience' sake, $\eta_{4} \equiv 1$. Eliminating $f_{1}, \cdots, f_{4}, g_{1}$, $\cdots, g_{4}$ from these relations, we get

$$
\operatorname{det}\left(a_{i}^{1}\left(\eta_{i}-x_{1}\right), a_{i}^{2}\left(\eta_{i}-x_{2}\right), a_{i}^{3}\left(\eta_{i} \eta_{1} \eta_{2}-x_{3}\right), \eta_{i} \eta_{3}-x_{4} ; 1 \leqq i \leqq 4\right) \equiv 0,
$$

which may be regarded as an identity with independent variables $\eta_{1}, \eta_{2}, \eta_{3}$. Substitute $\eta_{1}=\eta_{2}=\eta_{3}=1$. By the assumption for $a_{i}^{j}$, we obtain $x_{1}=1$, $x_{2}=1, x_{3}=1$ or $x_{4}=1$. Let $x_{1}=1$. If we put $\eta_{3}=\eta_{4}=1$, we see $x_{2}=1$ or $x_{4}=1$. For the case $x_{1}=x_{2}=1$, we get by substituting $\eta_{1}=1$ an absurd identity

$$
\left(a_{2}^{1} a_{3}^{2}-a_{2}^{2} a_{3}^{1}\right)\left(a_{1}^{3}-1\right)\left(\eta_{2}-1\right)\left(\eta_{3}-1\right)\left(\eta_{2}-x_{3}\right)\left(\eta_{3}-x_{4}\right)=0 .
$$

And, the case $x_{1}=x_{4}=1$ is reduced to the case $x_{1}=x_{2}=1$ by substituting $\eta_{3}=1$. Thus, the case $x_{1}=1$ does not occur. By the same argument, we can show that the case $x_{2}=1$ is also impossible. Moreover, the case $x_{3}=1$ and the case $x_{4}=1$ are reduced to the case $x_{1}=1$ or $x_{2}=1$ by substituting $\eta_{1}=\eta_{2}=1$ and $\eta_{1}=\eta_{3}=1$ respectively. Concludingly, there is no possibility of the case $(\varepsilon)$.

As was shown above, the case $(\zeta)$ only is possible. In this case $f=f_{1}: f_{2}: f_{3}: f_{4}$ and $g=g_{1}: g_{2}: g_{3}: g_{4}$ may be considered to be related as

$$
\begin{aligned}
& f_{i}=x_{i} \eta_{i}^{-1} g_{i} \\
& \sum_{j=1}^{4} a_{i}^{j} f_{j}=\eta_{i}\left(\sum_{j=1}^{4} a_{i}^{j} g_{j}\right) \quad(1 \leqq i \leqq 4)
\end{aligned}
$$

after changing indices, where $x_{1}, \cdots, x_{4} \in C^{*}, \eta_{1}, \eta_{2}, \eta_{3} \in H^{*}, t\left(\left[\eta_{1}\right],\left[\eta_{2}\right],\left[\eta_{3}\right]\right)$ $=3$ and $\eta_{4} \equiv 1$. As in the case $(\varepsilon)$, we have an identity

$$
\operatorname{det}\left(a_{i}^{j}\left(\eta_{i} \eta_{j}-x_{i}\right) ; 1 \leqq i, j \leqq 4\right) \equiv 0,
$$

with independent variables $\eta_{1}, \eta_{2}, \eta_{3}$ and we can conclude that

$$
x_{1}=x_{2}=x_{3}=1, \quad x_{4}=-1
$$

by substituting suitable particular values of $\eta_{1}, \eta_{2}, \eta_{3}$ into (7.7). Here, we can find constants $a_{i}^{i}$ such that (7.7) holds identically regarding $\eta_{1}, \eta_{2}, \eta_{3}$ 
as independent variables and any minor of the matrix $\left(a_{i}^{j}\right)$ does not vanish. And, for hyperplanes $H_{i}$ defined as (7.4) with these constants $a_{i}^{j}$ we can take two distinct algebraically non-degenerate meromorphic maps $f$ and $g$ such that $\nu\left(f, H_{i}\right)=\nu\left(g, H_{i}\right)(1 \leqq i \leqq 8)$. We note here the example for the particular case $N=3$ given in $\S 6.3$ is a special type of the case stated here. As is easily seen by (7.6), the set $V_{f, g}$ given in Definition 5.1 is included in an algebraic set

$$
\begin{array}{ll} 
& z_{i}\left(\sum_{j=1}^{4} a_{i}^{j} z_{j}\right)=w_{i}\left(\sum_{j=1}^{4} a_{i}^{j} w_{j}\right) \quad(i=1,2,3) \\
\tilde{V} ; \quad & z_{1}+z_{2}+z_{3}+z_{4}=w_{1}+w_{2}+w_{3}+w_{4} \\
& z_{4}=-w_{4},
\end{array}
$$

where $\left(z_{1}: z_{2}: z_{3}: z_{4}, w_{1}: w_{2}: w_{3}: w_{4}\right)$ is a system of homogeneous coordinates on $P^{3}(C) \times P^{3}(C)$. The author does not know geometric meanings of the condition (7.7) for constants $a_{i}^{j}$ and the algebric set $\tilde{V}$. Further studies in this direction are expected.

Added in proof: Recently, the author found a gap in the proof of Lemma 6.5. This is filled by the more precise study of possible types of $h_{i}$ 's. The details are to be published elsewhere.

\section{REFERENCES}

[1] E. Borel, Sur les zéros des fonctions entières, Acta Math., 20 (1897), 357-396.

[2] H. Fujimoto, On meromorphic maps into the complex projective space, J. Math. Soc. Japan 26 (1974), 272-288.

[ 3 ] H. Fujimoto, The uniqueness problem of meromorphic maps into the complex projective space, Nagoya Math. J., 58 (1975), 1-23.

[ 4 ] R. Nevanlinna, Einige Eindeutigkeitssätze in der Theorie der meromorphen Funktionen, Acta Math., 48 (1926), 367-391.

[ 5 ] G. Pólya, Bestimmung einer ganzen Funktionen endlichen Geschlechts durch viererlei Stellen, Math. Tidsskrift B, København 1921, 19-21.

Nagoya University 